\title{
Ulam stability of a alternate additive-quadratic functional equation in IFB space
}

\author{
Mohan Arunkumar ${ }^{1 *}$, Elumalai Sathya ${ }^{2}$ and Thirumal Namachivayam ${ }^{3}$
}

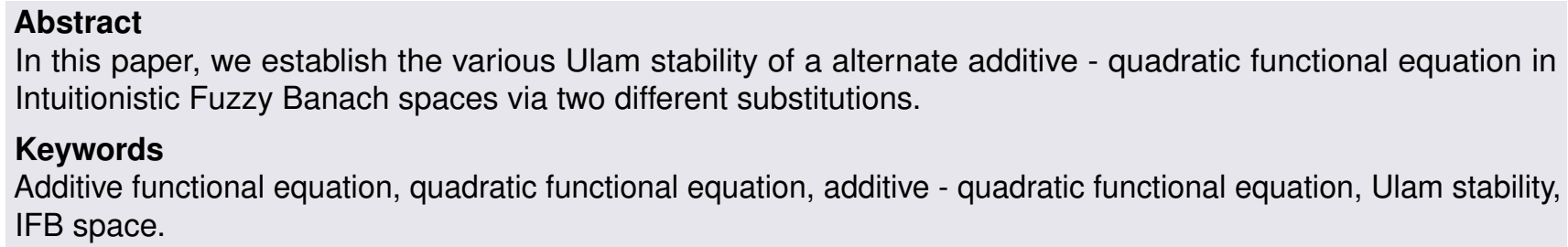

1,3 Department of Mathematics, Department of Mathematics, Government Arts College, Tiruvannamalai - 606 603, Tamil Nadu, India. ${ }^{2}$ Department of Mathematics, Shanmuga Industries Arts and Science College, Tiruvannamalai - 606 603, Tamil Nadu, India.

*Corresponding author: ${ }^{1}$ drarun4maths@gmail.com; ${ }^{2}$ sathya24mathematics@gmail.com; ${ }^{3}$ namachivayam1968@gmail.com

Article History: Received 22 November 2018; Accepted 09 May 2019

(C)2019 MJM.

\section{Contents}

1 Introduction 203

2

Basic Definitions of IFB Space. .204

3

4

IFBS: Stability Results: Substitution - 1 ........ 205

IFBS: Stability Results: Substitution - 2........210

References $. \ldots \ldots \ldots \ldots \ldots \ldots \ldots \ldots \ldots \ldots \ldots \ldots \ldots, 215$

\section{Introduction}

S.M. Ulam [43] is the pioneer for the stability problem in functional equations. In 1940, while he was delivering a talk before the Mathematics Club of University of Wisconsin, he discussed a number of unanswered problems. Among those was the following question concerning the stability of homomorphisms:

Let $G$ be a group and $G^{\prime}$ be a metric group with metric $\rho(.,$.$) . Given \varepsilon>0$ does there exist a $\delta>0$ such that if $a$ function $f: G \rightarrow G^{\prime}$ satisfies the inequality $\rho(f(x y), f(x) f(y))<$ $\delta$ for all $x, y \in G$, then there exists a homomorphism $h: G \rightarrow$ $G^{\prime}$ with $\rho(f(x), h(x))<\varepsilon$ for all $x \in G$ ?

In 1941, D.H. Hyers [22] gave a partial answer to the question of Ulam. In 1950, T. Aoki [2] and in 1978, Th.M. Rassias [36] explored the further generalization of Hyers theorem for linear mappings by considering an unbounded Cauchy difference for sum of powers of norms The result of Rassias has influenced the development of what is now called the Ulam -
Hyers - Rassias stability theory for functional equations.

In 1982, a similar stability theorem was proved by

J.M.Rassias [34] in which he replaced the term sum of powers of norms by product of norms. Later this stability result is called the Ulam - Gavruta - Rassias stability of functional equations.

All the above stability results are further generalized by P. Gavruta [19] in 1994 considering the control function as function of variables and proved the following theorem.

Theorem 1.1. [19] Let $(G,+)$ be an Abelian group, $(X,\|\cdot\|)$ be a Banach space and $\phi: G \times G \rightarrow[0, \infty)$ be a mapping such that

$$
\Phi(x, y)=\sum_{k=0}^{\infty} 2^{-k} \phi\left(2^{k} x, 2^{k} y\right)<\infty .
$$

If a function $f: G \rightarrow E$ satisfies the inequality

$$
\|f(x+y)-f(x)-f(y)\| \leq \phi(x, y)
$$

for any $x, y \in G$, then there exists a unique additive function $T: G \rightarrow E$ such that

$$
\|f(x)-T(x)\| \leq \frac{1}{2} \Phi(x, x)
$$

for all $x$ in $G$. If moreover $f(t x)$ is continuous in $t$ for each fixed $x \in G$, then $T$ is linear. 
This stability result is called Generalized Ulam - Hyers Rassias stability of functional equations.

Very recently J.M. Rassias [38] introduced a new concept on stability by introducing the mixed type product-sum of powers of norms. This stability result is called JMRassias stability of functional equations.

The solution and stability of following additive quadratic functional equations

$$
\begin{aligned}
& f(2 x+y)+f(2 x-y) \\
& =2 f(x+y)+2 f(x-y)+2 f(2 x)-4 f(x) \\
& f(2 x+y)+f(2 x-y) \\
& =f(x+y)+f(x-y)+2 f(2 x)-2 f(x) \\
& g(x+y)+g(x-y)=2 g(x)+g(y)+g(-y) \\
& f(x-t)+f(y-t)+f(z-t) \\
& =3 f\left(\frac{x+y+z}{3}-t\right)+f\left(\frac{2 x-y-z}{3}\right) \\
& +f\left(\frac{-x+2 y-z}{3}\right)+f\left(\frac{-x-y+2 z}{3}\right) \\
& f(x+2 y+3 z)+f(x-2 y+3 z) \\
& +f(x+2 y-3 z)+f(x-2 y-3 z) \\
& =4 f(x)+8[f(y)+f(-y)]+18[f(z)+f(-z)]
\end{aligned}
$$

were investigated by A. Najati, M.B.Moghimi [31], M.E. Gordji et. al., [18], M. J. Rassias et. al., [33], M.Arunkumar, J.M. Rassias [4] and M. Arunkumar, P. Agilan [5, 6].

In this paper, we establish the Ulam stability of a alternate additive - quadratic functional equation of the form

$$
\begin{aligned}
& h\left(z_{1}+2 z_{2}+3 z_{3}\right)-h\left(z_{1}-2 z_{2}+3 z_{3}\right) \\
& +h\left(z_{1}+2 z_{2}-3 z_{3}\right)-h\left(z_{1}-2 z_{2}-3 z_{3}\right) \\
& =8 h\left(z_{1}\right)-8 h\left(z_{1}-z_{2}\right)+4\left[h\left(z_{2}\right)+h\left(-z_{2}\right)\right]
\end{aligned}
$$

in Intuitionistic Fuzzy Banach spaces via two different substitutions.

\section{Basic Definitions of IFB Space}

Now, we recall the basic definitions and notations in the setting of intuitionistic fuzzy normed space which was introduced by Saadati and Park [39].

Definition 2.1. [39] A binary operation $*:[0,1] \times[0,1] \longrightarrow$ $[0,1]$ is said to be continuous $t$-norm if $*$ satisfies the following conditions:

(1) * is commutative and associative;

(2) $*$ is continuous;

(3) $a * 1=$ a for all $a \in[0,1]$;

(4) $a * b \leq c * d$ whenever $a \leq c$ and $b \leq d$ for all $a, b, c, d \in[0,1]$.
Definition 2.2. [39] A binary operation $\diamond:[0,1] \times[0,1] \longrightarrow$ $[0,1]$ is said to be continuous $t$-conorm if $\diamond$ satisfies the following conditions:

(1') $\diamond$ is commutative and associative;

$\left(2^{\prime}\right) \diamond$ is continuous;

(3') $a \diamond 0=$ a for all $a \in[0,1]$;

(4') $a \diamond b \leq c \diamond d$ whenever $a \leq c$ and $b \leq d$ for all $a, b, c, d \in[0,1]$.

Definition 2.3. [39] The five-tuple $(X, \mu, v, *, \diamond)$ is said to be an intuitionistic fuzzy normed space (for short, IFNS) if $\mathscr{A}$ is a vector space, $*$ is a continuous $t$-norm, $\diamond$ is a continuous $t-$ conorm, and $\mu, v$ are fuzzy sets on $X \times(0, \infty)$ satisfying the following conditions. For every $x, y \in \mathscr{A}$ and $s, t>0$

(IFN1) $\mu(x, t)+v(x, t) \leq 1$,

(IFN2) $\mu(x, t)>0$,

(IFN3) $\mu(x, t)=1$, if and only if $z=0$.

(IFN4) $\mu(\alpha x, t)=\mu\left(x, \frac{t}{\alpha}\right)$ for each $\alpha \neq 0$,

(IFN5) $\mu(x, t) * \mu(y, s) \leq \mu(x+y, t+s)$,

(IFN6) $\mu(x, \cdot):(0, \infty) \rightarrow[0,1]$ is continuous,

(IFN7) $\lim _{t \rightarrow \infty} \mu(x, t)=1$ and $\lim _{t \rightarrow 0} \mu(x, t)=0$,

(IFN8) $v(x, t)<1$,

(IFN9) $v(x, t)=0$, if and only if $z=0$.

(IFN10) $v(\alpha x, t)=v\left(x, \frac{t}{\alpha}\right)$ for each $\alpha \neq 0$,

(IFN11) $v(x, t) \diamond v(y, s) \geq v(x+y, t+s)$,

(IFN12) $v(x, \cdot):(0, \infty) \rightarrow[0,1]$ is continuous,

(IFN13) $\lim _{t \rightarrow \infty} v(x, t)=0$ and $\lim _{t \rightarrow 0} v(x, t)=1$.

In this case, $(\mu, v)$ is called an intuitionistic fuzzy norm.

Example 2.4. [39] Let $(X,\|\cdot\|)$ be a normed space. Let $a *$ $b=a b$ and $a \diamond b=\min \{a+b, 1\}$ for all $a, b \in[0,1]$. For all $x \in \mathscr{A}$ and every $t>0$, consider

$$
\mu(x, t)=\left\{\begin{array}{lll}
\frac{t}{t+\|x\|} & \text { if } & t>0 \\
0 & \text { if } & t \leq 0
\end{array}\right.
$$

and

$$
v(x, t)=\left\{\begin{array}{lll}
\frac{\|x\|}{t+\|x\|} & \text { if } & t>0 \\
0 & \text { if } & t \leq 0
\end{array}\right.
$$

Then $(X, \mu, v, *, \diamond)$ is an IFN-space. 
Definition 2.5. [39] Let $(X, \mu, v, *, \diamond)$ be an IFNS. Then, $a$ sequence $x=\left\{x_{k}\right\}$ is said to be intuitionistic fuzzy convergent to a point $L \in \mathscr{A}$ if

$\lim \mu\left(x_{k}-L, t\right)=1$ and $\lim v\left(x_{k}-L, t\right)=0$

for all $t>0$. In this case, we write

$$
x_{k} \stackrel{I F}{\longrightarrow} L \quad \text { as } \quad k \rightarrow \infty
$$

Definition 2.6. [39] Let $(X, \mu, v, *, \diamond)$ be an IFN-space. Then, $x=\left\{x_{k}\right\}$ is said to be intuitionistic fuzzy Cauchy sequence if

$$
\mu\left(x_{k+p}-x_{k}, t\right)=1 \quad \text { and } \quad v\left(x_{k+p}-x_{k}, t\right)=0
$$

for all $t>0$, and $p=1,2 \cdots$.

Definition 2.7. [39] Let $(X, \mu, v, *, \diamond)$ be an IFN-space. Then $(X, \mu, v, *, \diamond)$ is said to be complete if every intuitionistic fuzzy Cauchy sequence in $(X, \mu, v, *, \diamond)$ is intuitionistic fuzzy convergent $(X, \mu, v, *, \diamond)$.

Here and subsequently, assume that

- $\mathscr{A}$ - linear space;

- $\left(\mathscr{C}, \mu^{\prime}, v^{\prime}\right)$ - intuitionistic fuzzy normed space;

- $(\mathscr{B}, \mu, v)$ - intuitionistic fuzzy Banach space.

Now, we use the following notation for a given mapping $h: \mathscr{A} \longrightarrow \mathscr{B}$ such that

$$
\begin{aligned}
H\left(z_{1}, z_{2}, z_{3}\right)= & h\left(z_{1}+2 z_{2}+3 z_{3}\right)-h\left(z_{1}-2 z_{2}+3 z_{3}\right) \\
& +h\left(z_{1}+2 z_{2}-3 z_{3}\right)-h\left(z_{1}-2 z_{2}-3 z_{3}\right) \\
& -8 h\left(z_{1}\right)+8 h\left(z_{1}-z_{2}\right) \\
& -4\left[h\left(z_{2}\right)+h\left(-z_{2}\right)\right]
\end{aligned}
$$

for all $z_{1}, z_{2}, z_{3} \in \mathscr{A}$

\section{IFBS: Stability Results: Substitution - 1}

In this section, we investigate the generalized Ulam-Hyers stability of the functional equation (1.9) in IFB space.

Theorem 3.1. Let $\Delta \in\{1,-1\}$. Let $H: \mathscr{A} \rightarrow \mathscr{B}$ be an odd function satisfying the inequality

$$
\left.\begin{array}{l}
\mu\left(H\left(z_{1}, z_{2}, z_{3}\right), t\right) \geq \mu^{\prime}\left(\Omega_{\mu}\left(z_{1}, z_{2}, z_{3}\right), t\right) \\
\nu\left(H\left(z_{1}, z_{2}, z_{3}\right), t\right) \leq v^{\prime}\left(\Omega_{v}\left(z_{1}, z_{2}, z_{3}\right), t\right)
\end{array}\right\}
$$

for all $z_{1}, z_{2}, z_{3} \in \mathscr{A}$ and all $t>0$. Then there exists a unique additive mapping $\mathscr{H}_{1}: \mathscr{A} \longrightarrow \mathscr{B}$ satisfying (1.9) and

$$
\left.\begin{array}{c}
\mu\left(\mathscr{H}_{1}(z)-h(z), t\right) \geq \mu^{\prime}\left(\Omega_{\mu}(z, z, 0), t|3-p|\right) \\
v\left(\mathscr{H}_{1}(z)-h(z), t\right) \leq v^{\prime}\left(\Omega_{v}(z, z, 0), t|3-p|\right)
\end{array}\right\}
$$

for all $z \in \mathscr{A}$ and all $t>0$. Here $\Omega_{\mu}, \Omega_{v}: \mathscr{A} \times \mathscr{A} \times \mathscr{A} \longrightarrow \mathscr{C}$ are functions such that for some $0<\left(\frac{p}{3}\right)^{\Delta}<1$ with

$$
\left.\begin{array}{l}
\mu^{\prime}\left(\Omega_{\mu}\left(3^{n \Delta} z, 3^{n \Delta} z, 3^{n \Delta} z\right), t\right) \geq \mu^{\prime}\left(p^{n \Delta} \Omega_{\mu}(z, z, z), t\right) \\
v^{\prime}\left(\Omega_{v}\left(3^{n \Delta} z, 3^{n \Delta} z, 3^{n \Delta} z\right), t\right) \leq v^{\prime}\left(p^{n \Delta} \Omega_{v}(z, z, z), t\right)
\end{array}\right\}
$$

for all $z \in \mathscr{A}$ and all $t>0$ and

$$
\left.\begin{array}{l}
\lim _{n \rightarrow \infty} \mu^{\prime}\left(\Omega_{\mu}\left(3^{\Delta n} z_{1}, 3^{\Delta n} z_{2}, 3^{n \Delta} z_{3}\right), 3^{\Delta n} t\right)=1 \\
\lim _{n \rightarrow \infty} v^{\prime}\left(\Omega_{v}\left(3^{\Delta n} z_{1}, 3^{\Delta n} z_{2}, 3^{n \Delta} z_{3}\right), 3^{\Delta n} t\right)=0
\end{array}\right\}
$$

for all $z_{1}, z_{2}, z_{3} \in \mathscr{A}$ and all $t>0$.

Proof. Case (1):

Let $\Delta=1$. Using oddness of $h$ and transform $\left(z_{1}, z_{2}, z_{3}\right)$ by $(z, z, 0)$ in $(3.1)$, we have

$$
\left.\mu(h(3 z)-3 h(z), t) \geq \mu^{\prime}\left(\Omega_{\mu}(z, z, 0), t\right)\right\}
$$

for all $z \in \mathscr{A}$ and all $t>0$. Using (IFN4) and (IFN10) in (3.5), we arrive

$$
\left.\begin{array}{l}
\mu\left(\frac{h(3 z)}{3}-h(z), \frac{t}{3}\right) \geq \mu^{\prime}\left(\Omega_{\mu}(z, z, 0), t\right) \\
v\left(\frac{h(3 z)}{3}-h(z), \frac{t}{3}\right) \leq v^{\prime}\left(\Omega_{v}(z, z, 0), t\right)
\end{array}\right\}
$$

for all $z \in \mathscr{A}$ and all $t>0$. Substituting $z$ by $3^{n} z$ in (3.6), we have

$$
\left.\begin{array}{l}
\mu\left(\frac{h\left(3^{n+1} z\right)}{3}-h\left(3^{n} z\right), \frac{t}{3}\right) \geq \mu^{\prime}\left(\Omega_{\mu}\left(3^{n} z, 3^{n} z, 0\right), t\right) \\
\left.v\left(\frac{h\left(3^{n+1} z\right)}{3}-h\left(3^{n} z\right), \frac{t}{3}\right) \leq v^{\prime}\left(\Omega_{v}\left(3^{n} z, 3^{n} z, 0\right)\right), t\right)
\end{array}\right\}
$$

for all $z \in \mathscr{A}$ and all $t>0$. It is easy to verify from (3.7) and using (3.3), (IFN4), (IFN10) that

$$
\left.\begin{array}{l}
\mu\left(\frac{h\left(3^{n+1} z\right)}{3^{(n+1)}}-\frac{h\left(3^{n} z\right)}{3^{n}}, \frac{t}{3 \cdot 3^{n}}\right) \geq \mu^{\prime}\left(\Omega_{\mu}(z, z, 0), \frac{t}{p^{n}}\right) \\
\nu\left(\frac{h\left(3^{n+1} z\right)}{3^{(n+1)}}-\frac{h\left(3^{n} z\right)}{3^{n}}, \frac{}{3 \cdot 3^{n}}\right) \leq v^{\prime}\left(\Omega_{v}(z, z, 0), \frac{t}{p^{n}}\right)
\end{array}\right\}
$$

for all $z \in \mathscr{A}$ and all $t>0$. Interchanging $t$ into $p^{n} t$ in (3.8), we have

$$
\left.\begin{array}{l}
\mu\left(\frac{h\left(3^{n+1} z\right)}{3^{(n+1)}}-\frac{h\left(3^{n} z\right)}{3^{n}}, \frac{t \cdot p^{n}}{3 \cdot 3^{n}}\right) \geq \mu^{\prime}\left(\Omega_{\mu}(z, z, 0), t\right) \\
\nu\left(\frac{h\left(3^{n+1} z\right)}{3^{(n+1)}}-\frac{h\left(3^{n} z\right)}{3^{n}}, \frac{t \cdot p^{n}}{3 \cdot 3^{n}}\right) \leq v^{\prime}\left(\Omega_{\nu}(z, z, 0), t\right)
\end{array}\right\}
$$

for all $z \in \mathscr{A}$ and all $t>0$. It is easy to see that

$$
\frac{h\left(3^{n} z\right)}{3^{n}}-h(z)=\sum_{i=0}^{n-1} \frac{h\left(3^{i+1} z\right)}{3^{(i+1)}}-\frac{h\left(3^{i} z\right)}{3^{i}}
$$


for all $z \in \mathscr{A}$. From equations (3.8) and (3.10), we arrive

$$
\left.\begin{array}{c}
\mu\left(\frac{h\left(3^{n} z\right)}{3^{n}}-h(z), \sum_{i=0}^{n-1} \frac{t p^{i}}{3 \cdot 3^{i}}\right) \\
\quad \geq \prod_{i=0}^{n-1} \mu\left(\frac{h\left(3^{i+1} z\right)}{3^{(i+1)}}-\frac{h\left(3^{i} z\right)}{3^{i}}, \frac{t p^{i}}{3 \cdot 3^{i}}\right) \\
v\left(\frac{h\left(3^{n} z\right)}{3^{n}}-h(z), \sum_{i=0}^{n-1} \frac{t p^{i}}{3 \cdot 3^{i}}\right) \\
\quad \leq \prod_{i=0}^{n-1} v\left(\frac{h\left(3^{i+1} z\right)}{3^{(i+1)}}-\frac{h\left(3^{i} z\right)}{3^{i}}, \frac{t p^{i}}{3 \cdot 3^{i}}\right)
\end{array}\right\}
$$

with

$$
\prod_{i=0}^{n-1} c_{j}=c_{1} * c_{2} * \cdots * c_{n} \quad \text { and } \quad \prod_{i=0}^{n-1} d_{j}=d_{1} \diamond d_{2} \diamond \cdots \diamond d_{n}
$$

for all $z \in \mathscr{A}$ and all $t>0$. Thus from (3.9) and (3.11), we get

$$
\begin{aligned}
& \mu\left(\frac{h\left(3^{n} z\right)}{3^{n}}-h(z), \sum_{i=0}^{n-1} \frac{t p^{i}}{3 \cdot 3^{i}}\right) \\
& \geq \prod_{i=0}^{n-1} \mu^{\prime}\left(\Omega_{\mu}(z, z, 0), t\right)=\mu^{\prime}\left(\Omega_{\mu}(z, z, 0), t\right) \\
& v\left(\frac{h\left(3^{n} z\right)}{3^{n}}-h(z), \sum_{i=0}^{n-1} \frac{t p^{i}}{3 \cdot 3^{i}}\right) \\
& \leq \prod_{i=0}^{n-1} v^{\prime}\left(\Omega_{v}(z, z, 0), t\right)=v^{\prime}\left(\Omega_{v}(z, z, 0), t\right)
\end{aligned}
$$

for all $z \in \mathscr{A}$ and all $t>0$. Replacing $z$ by $3^{m} z$ in (3.12) and using (3.3), (IFN4), (IFN10), we obtain

$$
\begin{aligned}
& \mu\left(\frac{h\left(3^{n+m} z\right)}{3^{(n+m)}}-\frac{h\left(3^{m} z\right)}{3^{m}}, \sum_{i=0}^{n-1} \frac{t p^{i}}{3 \cdot 3^{(i+m)}}\right) \\
& \geq \mu^{\prime}\left(\Omega_{\mu}\left(3^{m} z, 3^{m} z, 0\right), t\right) \\
& =\mu^{\prime}\left(\Omega_{\mu}(z, z, 0), \frac{t}{p^{m}}\right) \\
& v\left(\frac{\left.h 3^{n+m} z\right)}{3^{(n+m)}}-\frac{h\left(3^{m} z\right)}{3^{m}}, \sum_{i=0}^{n-1} \frac{t p^{i}}{3 \cdot 3^{(i+m)}}\right) \\
& \leq v^{\prime}\left(\Omega_{v}\left(3^{m} z, 3^{m} z, 0\right), t\right) \\
& =v^{\prime}\left(\Omega_{v}(z, z, 0), \frac{t}{p^{m}}\right)
\end{aligned}
$$

for all $z \in \mathscr{A}$ and all $t>0$ and all $m, n \geq 0$. Interchanging $t$ by $p^{m} t$ in (3.13), we arrive

$$
\begin{aligned}
& \mu\left(\frac{h\left(3^{n+m} z\right)}{3^{(n+m)}}-\frac{h\left(3^{m} z\right)}{3^{m}}, \sum_{i=0}^{n-1} \frac{t p^{i+m}}{3 \cdot 3^{(i+m)}}\right) \\
& \geq \mu^{\prime}\left(\Omega_{\mu}(z, z, 0), t\right) \\
& v\left(\frac{\left.h 3^{n+m}\right)}{3^{(n+m)}}-\frac{h\left(3^{m} z\right)}{3^{m}}, \sum_{i=0}^{n-1} \frac{t p^{i+m}}{3 \cdot 3^{(i+m)}}\right) \\
& \leq v^{\prime}\left(\Omega_{v}(z, z, 0), t\right)
\end{aligned}
$$

for all $z \in \mathscr{A}$ and all $t>0$ and all $m, n \geq 0$. Using (IFN4), (IFN10) in (3.14) implies that

$$
\left.\begin{array}{l}
\mu\left(\frac{h\left(3^{n+m} z\right)}{3^{(n+m)}}-\frac{h\left(3^{m} z\right)}{3^{m}}, t\right) \geq \mu^{\prime}\left(\Omega_{\mu}(z, z, 0), \frac{t}{\sum_{i=m}^{n-1} \frac{p^{i}}{3 \cdot 3^{i}}}\right) \\
v\left(\frac{h\left(3^{n+m} z\right)}{3^{(n+m)}}-\frac{h\left(3^{m} z\right)}{3^{m}}, t\right) \leq \nu^{\prime}\left(\Omega_{v}(z, z, 0), \frac{t}{\sum_{i=m}^{n-1} \frac{p^{i}}{3 \cdot 3^{i}}}\right)
\end{array}\right\}
$$

holds for all $z \in \mathscr{A}$ and all $t>0$ and all $m, n \geq 0$. Since $0<p<1$ and $\sum_{i=0}^{n}\left(\frac{p}{1}\right)^{i}<\infty$. The Cauchy criterion for convergence in IFNS shows that the sequence $\left\{\frac{h\left(3^{n} z\right)}{3^{n}}\right\}$ is Cauchy in $(\mathscr{B}, \mu, v)$. Since $(\mathscr{B}, \mu, v)$ is a complete IFN-space this sequence converges to some point $\mathscr{H}_{1}(z) \in \mathscr{B}$. So, one can define the mapping $\mathscr{H}_{1}: \mathscr{A} \longrightarrow \mathscr{B}$ by

$\lim _{n \rightarrow \infty} \mu\left(\frac{h\left(3^{n} z\right)}{3^{n}}-\mathscr{H}_{1}(z), t\right)=1$, and

$\lim _{n \rightarrow \infty} v\left(\frac{h\left(3^{n} z\right)}{3^{n}}-\mathscr{H}_{1}(z), t\right)=0$ for all $z \in \mathscr{A}$ and all $t>0$. Hence

$$
\frac{h\left(3^{n} z\right)}{3^{n}} \stackrel{I F}{\longrightarrow} \mathscr{H}_{1}(z), \quad \text { as } \quad n \rightarrow \infty .
$$

Letting $m=0$ and $n$ tend to infinity in (3.15), we arrive

$$
\left.\begin{array}{l}
\mu\left(\mathscr{H}_{1}(z)-h(z), t\right) \geq \mu^{\prime}\left(\Omega_{\mu}(z, z, 0), t(3-p)\right) \\
v\left(\mathscr{H}_{1}(z)-h(z), t\right) \leq v^{\prime}\left(\Omega_{v}(z, z, 0), t(3-p)\right)
\end{array}\right\}
$$

for all $z \in \mathscr{A}$ and all $t>0$. To prove $\mathscr{A}$ satisfies (1.9), replacing $\left(z_{1}, z_{2}, z_{3}\right)$ by $\left(3^{n} z_{1}, 3^{n} z_{2}, 3^{n} z_{3}\right)$ in (3.1) respectively, we obtain

$$
\left.\begin{array}{c}
\mu\left(\frac{1}{3^{n}} H\left(3^{n} z_{1}, 3^{n} z_{2}, 3^{n} z_{3}\right), t\right) \\
\geq \mu^{\prime}\left(\Omega_{\mu}\left(3^{n} z_{1}, 3^{n} z_{2}, 3^{n} z_{3}\right), 3^{n} t\right) \\
v\left(\frac{1}{3^{n}} H\left(3^{n} z_{1}, 3^{n} z_{2}, 3^{n} z_{3}\right), t\right) \\
\leq v^{\prime}\left(\Omega_{v}\left(3^{n} z_{1}, 3^{n} z_{2}, 3^{n} z_{3}\right), t\right)
\end{array}\right\}
$$

for all $z \in \mathscr{A}$ and all $t>0$. Now,

$$
\begin{aligned}
& \mu\left(\mathscr{H}_{1}\left(z_{1}+2 z_{2}+3 z_{3}\right)-\mathscr{H}_{1}\left(z_{1}-2 z_{2}+3 z_{3}\right)\right. \\
&+\mathscr{H}_{1}\left(z_{1}+2 z_{2}-3 z_{3}\right)-\mathscr{H}_{1}\left(z_{1}-2 z_{2}-3 z_{3}\right) \\
&\left.-8 \mathscr{H}_{1}\left(z_{1}\right)+8 \mathscr{H}_{1}\left(z_{1}-z_{2}\right)-4\left[h\left(z_{2}\right)+h\left(-z_{2}\right)\right], t\right) \geq \\
& \mu\left.\mathscr{H}_{1}\left(z_{1}+2 z_{2}+3 z_{3}\right)-\frac{1}{3^{n}} h_{o}\left(3^{n}\left(z_{1}+2 z_{2}+3 z_{3}\right)\right), \frac{t}{8}\right) * \\
& \mu(-\left.\mathscr{H}_{1}\left(z_{1}-2 z_{2}+3 z_{3}\right)+\frac{1}{3^{n}} h_{o}\left(3^{n}\left(z_{1}-2 z_{2}+3 z_{3}\right)\right), \frac{t}{8}\right) * \\
& \mu\left(\mathscr{H}_{1}\left(z_{1}+2 z_{2}-3 z_{3}\right)-\frac{1}{3^{n}} h_{o}\left(3^{n}\left(z_{1}+2 z_{2}-3 z_{3}\right)\right), \frac{t}{8}\right) * \\
& \mu\left(-\mathscr{H}_{1}\left(z_{1}-2 z_{2}-3 z_{3}\right)-\frac{1}{3^{n}}+h_{o}\left(3^{n}\left(z_{1}-2 z_{2}-3 z_{3}\right)\right), \frac{t}{8}\right) * \\
& \mu\left(-8 \mathscr{H}_{1}\left(z_{1}\right)+\frac{8}{3^{n}} h_{o}\left(3^{n} z_{1}\right), \frac{t}{8}\right) * \\
& \mu\left(8 \mathscr{H}_{1}\left(z_{1}-z_{2}\right)-\frac{8}{3^{n}} h_{o}\left(3^{n}\left(z_{1}-z_{2}\right)\right), \frac{t}{8}\right) * \\
& \mu\left(-4\left[\mathscr{H}_{1}\left(z_{2}\right)+\mathscr{H}_{1}\left(-z_{2}\right)\right]\right. \\
&\left.\quad+\frac{4}{3^{n}}\left[h_{o}\left(3^{n} z_{2}\right)+h_{o}\left(-3^{n} z_{2}\right)\right], \frac{t}{8}\right) * \\
& \mu\left(\frac{1}{3^{n}} h_{o}\left(3^{n}\left(z_{1}+2 z_{2}+3 z_{3}\right)\right)-\frac{1}{3^{n}} h_{o}\left(3^{n}\left(z_{1}-2 z_{2}+3 z_{3}\right)\right)\right. \\
& \quad+\frac{1}{3^{n}} h_{o}\left(3^{n}\left(z_{1}+2 z_{2}-3 z_{3}\right)\right)-\frac{1}{3^{n}} h_{o}\left(3^{n}\left(z_{1}-2 z_{2}-3 z_{3}\right)\right) \\
& \quad-\frac{8}{3^{n}} h_{o}\left(3^{n} z_{1}\right)+\frac{8}{3^{n}} h_{o}\left(3^{n}\left(z_{1}-z_{2}\right)\right) \\
&\left.\quad-\frac{4}{3^{n}}\left[h_{o}\left(3^{n} z_{2}\right)+h_{o}\left(-3^{n} z_{2}\right)\right], \frac{t}{8}\right)
\end{aligned}
$$


and

$$
\begin{aligned}
& v\left(\mathscr{H}_{1}\left(z_{1}+2 z_{2}+3 z_{3}\right)-\mathscr{H}_{1}\left(z_{1}-2 z_{2}+3 z_{3}\right)\right. \\
& +\mathscr{H}_{1}\left(z_{1}+2 z_{2}-3 z_{3}\right)-\mathscr{H}_{1}\left(z_{1}-2 z_{2}-3 z_{3}\right) \\
& \left.-8 \mathscr{H}_{1}\left(z_{1}\right)+8 \mathscr{H}_{1}\left(z_{1}-z_{2}\right)-4\left[h\left(z_{2}\right)+h\left(-z_{2}\right)\right], t\right) \\
& \geq v\left(\mathscr{H}_{1}\left(z_{1}+2 z_{2}+3 z_{3}\right)\right. \\
& \left.-\frac{1}{3^{n}} h_{o}\left(3^{n}\left(z_{1}+2 z_{2}+3 z_{3}\right)\right), \frac{t}{8}\right) \diamond \\
& v\left(-\mathscr{H}_{1}\left(z_{1}-2 z_{2}+3 z_{3}\right)\right. \\
& \left.+\frac{1}{3^{n}} h_{o}\left(3^{n}\left(z_{1}-2 z_{2}+3 z_{3}\right)\right), \frac{t}{8}\right) \diamond \\
& v\left(\mathscr{H}_{1}\left(z_{1}+2 z_{2}-3 z_{3}\right)\right. \\
& \left.-\frac{1}{3^{n}} h_{o}\left(3^{n}\left(z_{1}+2 z_{2}-3 z_{3}\right)\right), \frac{t}{8}\right) \diamond \\
& v\left(-\mathscr{H}_{1}\left(z_{1}-2 z_{2}-3 z_{3}\right)\right. \\
& \left.-\frac{1}{3^{n}}+h_{o}\left(3^{n}\left(z_{1}-2 z_{2}-3 z_{3}\right)\right), \frac{t}{8}\right) \diamond \\
& v\left(-8 \mathscr{H}_{1}\left(z_{1}\right)+\frac{8}{3^{n}} h_{o}\left(3^{n} z_{1}\right), \frac{t}{8}\right) \diamond \\
& v\left(8 \mathscr{H}_{1}\left(z_{1}-z_{2}\right)-\frac{8}{3^{n}} h_{o}\left(3^{n}\left(z_{1}-z_{2}\right)\right), \frac{t}{8}\right) \diamond \\
& v\left(-4\left[\mathscr{H}_{1}\left(z_{2}\right)+\mathscr{H}_{1}\left(-z_{2}\right)\right]\right. \\
& \left.+\frac{4}{3^{n}}\left[h_{o}\left(3^{n} z_{2}\right)+h_{o}\left(-3^{n} z_{2}\right)\right], \frac{t}{8}\right) \diamond \\
& v\left(\frac{1}{3^{n}} h_{o}\left(3^{n}\left(z_{1}+2 z_{2}+3 z_{3}\right)\right)\right. \\
& -\frac{1}{3^{n}} h_{o}\left(3^{n}\left(z_{1}-2 z_{2}+3 z_{3}\right)\right) \\
& +\frac{1}{3^{n}} h_{o}\left(3^{n}\left(z_{1}+2 z_{2}-3 z_{3}\right)\right) \\
& -\frac{1}{3^{n}} h_{o}\left(3^{n}\left(z_{1}-2 z_{2}-3 z_{3}\right)\right) \\
& -\frac{8}{3^{n}} h_{o}\left(3^{n} z_{1}\right)+\frac{8}{3^{n}} h_{o}\left(3^{n}\left(z_{1}-z_{2}\right)\right) \\
& \left.-\frac{4}{3^{n}}\left[h_{o}\left(3^{n} z_{2}\right)+h_{o}\left(-3^{n} z_{2}\right)\right], \frac{t}{8}\right)
\end{aligned}
$$

or all $z_{1}, z_{2}, z_{3} \in \mathscr{A}$ and all $t>0$. Letting $n \rightarrow \infty$ in (3.17), we arrive

$$
\left.\begin{array}{l}
\lim _{n \rightarrow \infty} \mu\left(\frac{1}{3^{n}} H\left(3^{n} z_{1}, 3^{n} z_{2}, 3^{n} z_{3}\right), \frac{t}{6}\right)=1 \\
\lim _{n \rightarrow \infty} v\left(\frac{1}{3^{n}} H\left(3^{n} z_{1}, 3^{n} z_{2}, 3^{n} z_{3}\right), \frac{t}{6}\right)=0
\end{array}\right\}
$$

for all $z \in \mathscr{A}$ and all $t>0$. Letting $n \rightarrow \infty$ in (3.18), (3.19) and using (3.20), we observe that $\mathscr{H}_{1}$ fulfills (1.9). Therefore, $\mathscr{H}_{1}$ is a additive mapping. In order to prove $\mathscr{H}_{1}(z)$ is unique, let $\mathscr{H}_{1}^{\prime}(z)$ be another additive functional equation satisfying
(1.9) and (3.2). Hence,

$$
\begin{aligned}
& \mu\left(\mathscr{H}_{1}(z)-\mathscr{H}_{1}^{\prime}(z), t\right) \\
& \geq \mu\left(\mathscr{H}_{1}\left(3^{n} z\right)-h\left(3^{n} z\right), \frac{t \cdot 3^{n}}{2}\right) * \\
& \mu\left(h\left(3^{n} z\right)-\mathscr{H}_{1}^{\prime}\left(3^{n} z\right), \frac{t \cdot 3^{n}}{2}\right) \\
& \geq \mu^{\prime}\left(\Omega_{\mu}(z, z, 0)\left(3^{n} z\right), \frac{t 3^{n}(3-p)}{2}\right) \\
& \geq \mu^{\prime}\left(\Omega_{\mu}(z, z, 0), \frac{t 3^{n}(3-p)}{2 \cdot p^{n}}\right) \\
& v\left(\mathscr{H}_{1}(z)-\mathscr{H}_{1}^{\prime}(z), t\right) \\
& \leq v\left(\mathscr{H}_{1}\left(3^{n} z\right)-h\left(3^{n} z\right), \frac{t \cdot 3^{n}}{2}\right) \diamond \\
& v\left(h\left(3^{n} z\right)-\mathscr{H}_{1}^{\prime}\left(3^{n} z\right), \frac{t \cdot 3^{n}}{2}\right) \\
& \leq v^{\prime}\left(\Omega_{v}(z, z, 0)\left(3^{n} z\right), \frac{t 3^{n}(3-p)}{2}\right) \\
& \leq v^{\prime}\left(\Omega_{v}(z, z, 0), \frac{t 3^{n}(3-p)}{2 \cdot p^{n}}\right)
\end{aligned}
$$

for all $z \in \mathscr{A}$ and all $t>0$.

Since $\lim _{n \rightarrow \infty} \frac{t 3^{n}(3-p)}{2 p^{n}}=\infty$, we obtain

$$
\left.\begin{array}{l}
\lim _{n \rightarrow \infty} \mu^{\prime}\left(\Omega_{\mu}(z), \frac{t 3^{n}(3-p)}{2 p^{n}}\right)=1 \\
\lim _{n \rightarrow \infty} v^{\prime}\left(\Omega_{v}(z), \frac{t 3^{n}(3-p)}{2 p^{n}}\right)=0
\end{array}\right\}
$$

for all $z \in \mathscr{A}$ and all $t>0$. Thus

$$
\left.\begin{array}{l}
\mu\left(\mathscr{H}_{1}(z)-\mathscr{H}_{1}^{\prime}(z), t\right)=1 \\
v\left(\mathscr{H}_{1}(z)-\mathscr{H}_{1}^{\prime}(z), t\right)=0
\end{array}\right\}
$$

for all $z \in \mathscr{A}$ and all $t>0$. Hence, $\mathscr{H}_{1}(z)=\mathscr{H}_{1}^{\prime}(z)$. Therefore, $\mathscr{H}_{1}(z)$ is unique.

Case 2:

For $\Delta=-1$. Putting $z$ by $\frac{z}{3}$ in (3.5), we get

$$
\left.\begin{array}{l}
\mu\left(h(z)-3 h\left(\frac{z}{3}\right), t\right) \geq \mu^{\prime}\left(\Omega_{\mu}\left(\frac{z}{3}, \frac{z}{3}, 0\right), t\right) \\
v\left(h(z)-3 h\left(\frac{z}{3}\right), t\right) \leq v^{\prime}\left(\Omega_{v}\left(\frac{z}{3}, \frac{z}{3}, 0\right), t\right)
\end{array}\right\}
$$

for all $z \in \mathscr{A}$ and all $t>0$. The rest of the proof is similar to that of Case 1. This completes the proof.

The following corollary is an immediate consequence of Theorem 3.1, regarding some stabilities of (1.9). 
Corollary 3.2. Assume an odd function $H: \mathscr{A} \longrightarrow \mathscr{B}$ satisfies the double inequality

$$
\begin{aligned}
& \mu\left(H\left(z_{1}, z_{2}, z_{3}\right), t\right) \\
& \geq\left\{\begin{array}{l}
\mu^{\prime}(\lambda, t), \\
\mu^{\prime}\left(\lambda \sum_{i=1}^{3}\left\|z_{i}\right\|^{a}, t\right), \\
\mu^{\prime}\left(\lambda \sum_{i=1}^{3}\left\|z_{i}\right\|^{a_{i}}, t\right),
\end{array}\right. \\
& v\left(H\left(z_{1}, z_{2}, z_{3}\right), t\right) \\
& \leq\left\{\begin{array}{l}
v^{\prime}(\lambda, t), \\
v^{\prime}\left(\lambda \sum_{i=1}^{3}\left\|z_{i}\right\|^{a}, t\right), \\
v^{\prime}\left(\lambda \sum_{i=1}^{3}\left\|z_{i}\right\|^{a_{i}}, t\right),
\end{array}\right.
\end{aligned}
$$

for all $z_{1}, z_{2}, z_{3} \in \mathscr{A}$ and all $t>0$, where $\lambda, a, a_{i}^{\prime}$ s are constants with $\lambda>0$. Then there exists a unique additive mapping $\mathscr{H}_{1}: \mathscr{A} \longrightarrow \mathscr{B}$ such that

$$
\begin{aligned}
& \mu\left(h(z)-\mathscr{H}_{1}(z), t\right) \\
& \geq\left\{\begin{array}{l}
\mu^{\prime}(\lambda,|3-p| t), \\
\mu^{\prime}\left(2 \lambda|| z||^{a}, t|3-p|\right), \\
\mu^{\prime}\left(\lambda\left[|| z||^{a_{1}}+\| z||^{a_{2}}\right], t|3-p|\right),
\end{array}\right. \\
& v\left(h(z)-\mathscr{H}_{1}(z), t\right) \\
& \quad \leq\left\{\begin{array}{l}
v^{\prime}(\lambda,|3-p| t), \\
v^{\prime}\left(2 \lambda|| z \|^{a}, t|3-p|\right), \\
v^{\prime}\left(\lambda\left[|| z||^{a_{1}}+|| z||^{a_{2}}\right], t|3-p|\right),
\end{array}\right.
\end{aligned}
$$

for all $z \in \mathscr{A}$ and all $t>0$.

Theorem 3.3. Let $\Delta \in\{1,-1\}$. Let $H: \mathscr{A} \rightarrow \mathscr{B}$ be an even function satisfying the inequality

$$
\left.\begin{array}{l}
\mu\left(H\left(z_{1}, z_{2}, z_{3}\right), t\right) \geq \mu^{\prime}\left(\Omega_{\mu}\left(z_{1}, z_{2}, z_{3}\right), t\right) \\
v\left(H\left(z_{1}, z_{2}, z_{3}\right), t\right) \leq v^{\prime}\left(\Omega_{v}\left(z_{1}, z_{2}, z_{3}\right), t\right)
\end{array}\right\}
$$

for all $z_{1}, z_{2}, z_{3} \in \mathscr{A}$ and all $t>0$. Then there exists a unique quadratic mapping $\mathscr{H}_{2}: \mathscr{A} \longrightarrow \mathscr{B}$ satisfying (1.9) and

$$
\left.\begin{array}{l}
\mu\left(\mathscr{H}_{2}(z)-h(z), t\right) \geq \mu^{\prime}\left(\Omega_{\mu}(z, z, 0), t|9-p|\right) \\
v\left(\mathscr{H}_{2}(z)-h(z), t\right) \leq v^{\prime}\left(\Omega_{v}(z, z, 0), t|9-p|\right)
\end{array}\right\}
$$

for all $z \in \mathscr{A}$ and all $t>0$. Here $\Omega_{\mu}, \Omega_{v}: \mathscr{A} \times \mathscr{A} \times \mathscr{A} \longrightarrow \mathscr{C}$ are functions such that for some $0<\left(\frac{p}{3}\right)^{\Delta}<1$ with

$$
\left.\begin{array}{l}
\mu^{\prime}\left(\Omega_{\mu}\left(3^{n \Delta} z, 3^{n \Delta} z, 3^{n \Delta} z\right), t\right) \geq \mu^{\prime}\left(p^{n \Delta} \Omega_{\mu}(z, z, z), t\right) \\
v^{\prime}\left(\Omega_{v}\left(3^{n \Delta} z, 3^{n \Delta} z, 3^{n \Delta} z\right), t\right) \leq v^{\prime}\left(p^{n \Delta} \Omega_{v}(z, z, z), t\right)
\end{array}\right\}
$$

for all $z \in \mathscr{A}$ and all $t>0$ and

$$
\left.\begin{array}{l}
\lim _{n \rightarrow \infty} \mu^{\prime}\left(\Omega_{\mu}\left(3^{\Delta n} z_{1}, 3^{\Delta n} z_{2}, 3^{n \Delta} z_{3}\right), 9^{\Delta n} t\right)=1 \\
\lim _{n \rightarrow \infty} v^{\prime}\left(\Omega_{v}\left(3^{\Delta n} z_{1}, 3^{\Delta n} z_{2}, 3^{n \Delta} z_{3}\right), 9^{\Delta n} t\right)=0
\end{array}\right\}
$$

for all $z_{1}, z_{2}, z_{3} \in \mathscr{A}$ and all $t>0$.
Proof. Case (1): Let $\Delta=1$.

Using evenness of $h$ and transform $\left(z_{1}, z_{2}, z_{3}\right)$ by $(z, z, 0)$ in (3.24), we have

$$
\left.\begin{array}{l}
\mu\left(h_{e}(3 x)-9 h_{e}(z), t\right) \geq \mu^{\prime}\left(\Omega_{\mu}(z, z, 0), t\right) \\
v\left(h_{e}(3 x)-9 h_{e}(z), t\right) \leq v^{\prime}\left(\Omega_{v}(z, z, 0), t\right)
\end{array}\right\}
$$

for all $z \in \mathscr{A}$ and all $t>0$. Using (IFN4) and (IFN10) in (3.28), we arrive

$$
\left.\begin{array}{l}
\mu\left(\frac{h(3 z)}{9}-h(z), \frac{t}{9}\right) \geq \mu^{\prime}\left(\Omega_{\mu}(z, z, 0), t\right) \\
v\left(\frac{h(3 z)}{9}-h(z), \frac{t}{9}\right) \leq v^{\prime}\left(\Omega_{v}(z, z, 0), t\right)
\end{array}\right\}
$$

for all $z \in \mathscr{A}$ and all $t>0$. The rest of the proof is similar to that of Theorem 3.1 .

The following corollary is an immediate consequence of Theorem 3.3, regarding some stabilities of (1.9).

Corollary 3.4. Assume an even function $H: \mathscr{A} \longrightarrow \mathscr{B}$ satisfies the double inequality

$$
\begin{aligned}
& \mu\left(H\left(z_{1}, z_{2}, z_{3}\right), t\right) \geq\left\{\begin{array}{l}
\mu^{\prime}(\lambda, t), \\
\mu^{\prime}\left(\lambda \sum_{i=1}^{3}\left\|z_{i}\right\|^{a}, t\right), \\
\mu^{\prime}\left(\lambda \sum_{i=1}^{3}\left\|z_{i}\right\|^{a_{i}}, t\right), \\
v^{\prime}(\lambda, t), \\
v^{\prime}\left(\lambda \sum_{i=1}^{3}\left\|z_{i}\right\|^{a}, t\right), \\
v^{\prime}\left(\lambda \sum_{i=1}^{3}\left\|z_{i}\right\|^{a_{i}}, t\right),
\end{array}\right\} \\
& v\left(H\left(z_{1}, z_{2}, z_{3}\right), t\right) \leq
\end{aligned}
$$

for all $z_{1}, z_{2}, z_{3} \in \mathscr{A}$ and all $t>0$, where $\lambda, a, a_{i}^{\prime}$ s are constants with $\lambda>0$. Then there exists a unique quadratic mapping $\mathscr{H}_{2}: \mathscr{A} \longrightarrow \mathscr{B}$ such that

$$
\begin{aligned}
& \mu\left(h(z)-\mathscr{H}_{2}(z), t\right) \\
& \geq\left\{\begin{array}{l}
\mu^{\prime}(\lambda,|9-p| t), \\
\mu^{\prime}\left(2 \lambda|| z||^{a}, t|9-p|\right), \\
\mu^{\prime}\left(\lambda\left[|| z||^{a_{1}}+|| z||^{a_{2}}\right], t|9-p|\right),
\end{array}\right. \\
& v\left(h(z)-\mathscr{H}_{2}(z), t\right) \\
& \leq\left\{\begin{array}{l}
v^{\prime}(\lambda,|9-p| t), \\
v^{\prime}\left(\left.\lambda|| z||\right|^{a}, t|9-p|\right), \\
v^{\prime}\left(\lambda\left[\left\|z||^{a_{1}}+\right\| z \|^{a_{2}}\right], t|9-p|\right)
\end{array}\right.
\end{aligned}
$$

for all $z \in \mathscr{A}$ and all $t>0$.

Theorem 3.5. Let $\Delta \in\{1,-1\}$. Let $H: \mathscr{A} \rightarrow \mathscr{B}$ be a function satisfying the inequality

$$
\left.\begin{array}{l}
\mu\left(H\left(z_{1}, z_{2}, z_{3}\right), t\right) \geq \mu^{\prime}\left(\Omega_{\mu}\left(z_{1}, z_{2}, z_{3}\right), t\right) \\
v\left(H\left(z_{1}, z_{2}, z_{3}\right), t\right) \leq v^{\prime}\left(\Omega_{v}\left(z_{1}, z_{2}, z_{3}\right), t\right)
\end{array}\right\}
$$

for all $z_{1}, z_{2}, z_{3} \in \mathscr{A}$ and all $t>0$. Then there exists a unique additive mapping $\mathscr{H}_{1}: \mathscr{A} \longrightarrow \mathscr{B}$ and a unique quadratic 
mapping $\mathscr{H}_{2}: \mathscr{A} \longrightarrow \mathscr{B}$ satisfying (1.9) and

$$
\begin{gathered}
\mu\left(h(z)-\mathscr{H}_{1}(z)-\mathscr{H}_{2}(z), 2 t\right) \\
\geq \mu^{\prime}\left(\Omega_{\mu}(z, z, 0), t|3-p|\right) * \\
\mu^{\prime}\left(\Omega_{\mu}(z, z, 0), t|3-p|\right) * \\
\mu^{\prime}\left(\Omega_{\mu}(-z,-z, 0), t|3-p|\right) * \\
\mu^{\prime}\left(\Omega_{\mu}(-z,-z, 0), t|3-p|\right) * \\
\mu^{\prime}\left(\Omega_{\mu}(z, z, 0), t|9-p|\right) * \\
\mu^{\prime}\left(\Omega_{\mu}(z, z, 0), t|9-p|\right) * \\
\mu^{\prime}\left(\Omega_{\mu}(-z,-z, 0), t|9-p|\right) * \\
\mu^{\prime}\left(\Omega_{\mu}(-z,-z, 0), t|9-p|\right) \\
v\left(h(z)-\mathscr{H}_{1}(z)-\mathscr{H}_{2}(z), 2 t\right) \\
\leq v^{\prime}\left(\Omega_{v}(z, z, 0), t|3-p|\right) \diamond \\
v^{\prime}\left(\Omega_{v}(z, z, 0), t|3-p|\right) \diamond \\
v^{\prime}\left(\Omega_{v}(-z,-z, 0), t|3-p|\right) \diamond \\
v^{\prime}\left(\Omega_{v}(-z,-z, 0), t|3-p|\right) \diamond \\
v^{\prime}\left(\Omega_{v}(z, z, 0), t|9-p|\right) \diamond \\
v^{\prime}\left(\Omega_{v}(z, z, 0), t|9-p|\right) \diamond \\
v^{\prime}\left(\Omega_{v}(-z,-z, 0), t|9-p|\right) \diamond \\
v^{\prime}\left(\Omega_{v}(-z,-z, 0), t|9-p|\right)
\end{gathered}
$$

for all $z \in \mathscr{A}$ and all $t>0$. Here $\Omega_{\mu}, \Omega_{v}: \mathscr{A} \times \mathscr{A} \times \mathscr{A} \longrightarrow \mathscr{C}$ are functions such that for some $0<\left(\frac{p}{3}\right)^{\Delta}<1 ; 0<\left(\frac{p}{4}\right)^{\Delta}<$ 1 with conditions (3.3), (3.26) and (3.4), (3.27) for all $z \in \mathscr{A}$ and all $t>0$ and for all $z_{1}, z_{2}, z_{3} \in \mathscr{A}$ and all $t>0$.

Proof. Let $h_{O}(z)=\frac{h(z)-h(-z)}{2}$ for all $z \in \mathscr{A}$. It is easy to verify that $h_{O}(0)=0$ and $h_{O}(-z)=-h_{O}(z)$ for all $z \in \mathscr{A}$. By definition of $h_{O}(z)$, we have

$$
\begin{aligned}
& \mu\left(h_{O}\left(z_{1}, z_{2}, z_{3}\right), t\right) \\
& =\mu\left(h\left(z_{1}, z_{2}, z_{3}\right)-h\left(-z_{1},-z_{2},-z_{3}\right), 2 t\right) \\
& \geq \mu\left(h\left(z_{1}, z_{2}, z_{3}\right), t\right) * \\
& \mu\left(h\left(-z_{1},-z_{2},-z_{3}\right), t\right) \\
& \geq \mu^{\prime}\left(\Omega_{\mu}\left(z_{1}, z_{2}, z_{3}\right), t\right) * \\
& \mu^{\prime}\left(\Omega_{\mu}\left(-z_{1},-z_{2},-z_{3}\right), t\right) \\
& v\left(h_{O}\left(z_{1}, z_{2}, z_{3}\right), t\right) \\
& =v\left(h\left(z_{1}, z_{2}, z_{3}\right)-h\left(-z_{1},-z_{2},-z_{3}\right), 2 t\right) \\
& \leq v\left(h\left(z_{1}, z_{2}, z_{3}\right), t\right) \diamond \\
& \quad v\left(h\left(-z_{1},-z_{2},-z_{3}\right), t\right) \\
& \leq v^{\prime}\left(\Omega_{v}\left(z_{1}, z_{2}, z_{3}\right), t\right) \diamond \\
& v^{\prime}\left(\Omega_{v}\left(-z_{1},-z_{2},-z_{3}\right), t\right)
\end{aligned}
$$

for all $z_{1}, z_{2}, z_{3} \in \mathscr{A}$ and all $t>0$. By Theorem 3.1, there exists a unique additive mapping $\mathscr{H}_{1}: \mathscr{A} \longrightarrow \mathscr{B}$ satisfying
(1.9) and

$$
\begin{gathered}
\mu\left(h_{O}(z)-\mathscr{H}_{1}(z), t\right) \\
\geq \mu^{\prime}\left(\Omega_{\mu}(z, z, 0), t|3-p|\right) * \\
\mu^{\prime}\left(\Omega_{\mu}(z, z, 0), t|3-p|\right) * \\
\mu^{\prime}\left(\Omega_{\mu}(-z,-z, 0), t|3-p|\right) * \\
\mu^{\prime}\left(\Omega_{\mu}(-z,-z, 0), t|3-p|\right) \\
v\left(h_{O}(z)-\mathscr{H}_{1}(z), t\right) \\
\leq v^{\prime}\left(\Omega_{v}(z, z, 0), t|3-p|\right) \diamond \\
v^{\prime}\left(\Omega_{v}(z, z, 0), t|3-p|\right) \diamond \\
v^{\prime}\left(\Omega_{v}(-z,-z, 0), t|3-p|\right) \diamond \\
v^{\prime}\left(\Omega_{v}(-z,-z, 0), t|3-p|\right)
\end{gathered}
$$

for all $z \in \mathscr{A}$ and all $t>0$.

Also, let $h_{E}(z)=\frac{h(z)+h(-z)}{2}$ for all $z \in \mathscr{A}$. It is easy to verify that $h_{E}(0)=0$ and $h_{E}(-z)=h_{E}(z)$ for all $z \in \mathscr{A}$. By definition of $h_{E}(z)$, we have

$$
\left.\begin{array}{l}
\mu\left(h_{E}\left(z_{1}, z_{2}, z_{3}\right), t\right) \\
=\mu\left(h\left(z_{1}, z_{2}, z_{3}\right)+h\left(-z_{1},-z_{2},-z_{3}\right), 2 t\right) \\
\geq \mu\left(h\left(z_{1}, z_{2}, z_{3}\right), t\right) * \mu\left(h\left(-z_{1},-z_{2},-z_{3}\right), t\right) \\
\geq \mu^{\prime}\left(\Omega_{\mu}\left(z_{1}, z_{2}, z_{3}\right), t\right) * \mu^{\prime}\left(\Omega_{\mu}\left(-z_{1},-z_{2},-z_{3}\right), t\right) \\
v\left(h_{E}\left(z_{1}, z_{2}, z_{3}\right), t\right) \\
=v\left(h\left(z_{1}, z_{2}, z_{3}\right)+h\left(-z_{1},-z_{2},-z_{3}\right), 2 t\right) \\
\leq v\left(h\left(z_{1}, z_{2}, z_{3}\right), t\right) \diamond v\left(h\left(-z_{1},-z_{2},-z_{3}\right), t\right) \\
\leq v^{\prime}\left(\Omega_{v}\left(z_{1}, z_{2}, z_{3}\right), t\right) \diamond v^{\prime}\left(\Omega_{v}\left(-z_{1},-z_{2},-z_{3}\right), t\right)
\end{array}\right\}
$$

for all $z_{1}, z_{2}, z_{3} \in \mathscr{A}$ and all $t>0$. Also, by Theorem 3.3, there exists a unique quadratic mapping $\mathscr{H}_{2}: \mathscr{A} \longrightarrow \mathscr{B}$ satisfying (1.9) and

$$
\left.\begin{array}{l}
\mu\left(h_{E}(z)-\mathscr{H}_{2}(z), t\right) \\
\geq \mu^{\prime}\left(\Omega_{\mu}(z, z, 0), t|9-p|\right) * \mu^{\prime}\left(\Omega_{\mu}(z, z, 0), t|9-p|\right) * \\
\mu^{\prime}\left(\Omega_{\mu}(-z,-z, 0), t|9-p|\right) * \mu^{\prime}\left(\Omega_{\mu}(-z,-z, 0), t|9-p|\right) \\
v\left(h_{E}(z)-\mathscr{H}_{2}(z), t\right) \\
\leq v^{\prime}\left(\Omega_{v}(z, z, 0), t|9-p|\right) \diamond v^{\prime}\left(\Omega_{v}(z, z, 0), t|9-p|\right) \diamond \\
v^{\prime}\left(\Omega_{v}(-z,-z, 0), t|9-p|\right) \diamond v^{\prime}\left(\Omega_{v}(-z,-z, 0), t|9-p|\right)
\end{array}\right\}
$$

for all $z \in \mathscr{A}$ and all $t>0$.

Suppose if we define a function $h(z)$ by

$$
h(z)=h_{O}(z)+h_{E}(z)
$$


for all $z \in \mathscr{A}$. It follows from (3.35), (3.37), (3.38), we arrive

$$
\begin{gathered}
\mu\left(h(z)-\mathscr{H}_{1}(z)-\mathscr{H}_{2}(z), 2 t\right) \\
\geq \mu\left(h_{O}(z)-\mathscr{H}_{1}(z), t\right) * \mu\left(h_{E}(z)-\mathscr{H}_{2}(z), t\right) \\
\geq \mu^{\prime}\left(\Omega_{\mu}(z, z, 0), t|3-p|\right) * \\
\mu^{\prime}\left(\Omega_{\mu}(z, z, 0), t|3-p|\right) * \\
\mu^{\prime}\left(\Omega_{\mu}(-z,-z, 0), t|3-p|\right) * \\
\mu^{\prime}\left(\Omega_{\mu}(-z,-z, 0), t|3-p|\right) * \\
\mu^{\prime}\left(\Omega_{\mu}(z, z, 0), t|9-p|\right) * \\
\mu^{\prime}\left(\Omega_{\mu}(z, z, 0), t|9-p|\right) * \\
\mu^{\prime}\left(\Omega_{\mu}(-z,-z, 0), t|9-p|\right) * \\
\mu^{\prime}\left(\Omega_{\mu}(-z,-z, 0), t|9-p|\right) \\
v\left(h(z)-\mathscr{H}_{1}(z)-\mathscr{H}_{2}(z), 2 t\right) \\
\leq v\left(h_{O}(z)-\mathscr{H}_{1}(z), t\right) \diamond v\left(h_{E}(z)-\mathscr{H}_{2}(z), t\right) \\
\leq v^{\prime}\left(\Omega_{v}(z, z, 0), t|3-p|\right) \diamond \\
v^{\prime}\left(\Omega_{v}(z, z, 0), t|3-p|\right) \diamond \\
v^{\prime}\left(\Omega_{v}(-z,-z, 0), t|3-p|\right) \diamond \\
v^{\prime}\left(\Omega_{v}(-z,-z, 0), t|3-p|\right) \diamond \\
v^{\prime}\left(\Omega_{v}(z, z, 0), t|9-p|\right) \diamond \\
v^{\prime}\left(\Omega_{v}(z, z, 0), t|9-p|\right) \diamond \\
v^{\prime}\left(\Omega_{v}(-z,-z, 0), t|9-p|\right) \diamond \\
v^{\prime}\left(\Omega_{v}(-z,-z, 0), t|9-p|\right)
\end{gathered}
$$

for all $z \in \mathscr{A}$ and all $t>0$.

The following corollary is an immediate consequence of Theorem 3.5, regarding some stabilities of (1.9).

Corollary 3.6. Assume a function $H: \mathscr{A} \longrightarrow \mathscr{B}$ satisfies the double inequality

$$
\begin{aligned}
& \mu\left(H\left(z_{1}, z_{2}, z_{3}\right), t\right) \geq\left\{\begin{array}{l}
\mu^{\prime}(\lambda, t), \\
\mu^{\prime}\left(\lambda \sum_{i=1}^{3}\left\|z_{i}\right\|^{a}, t\right), \\
\mu^{\prime}\left(\lambda \sum_{i=1}^{3}\left\|z_{i}\right\|^{a_{i}}, t\right),
\end{array}\right. \\
& v\left(H\left(z_{1}, z_{2}, z_{3}\right), t\right) \leq\left\{\begin{array}{l}
v^{\prime}(\lambda, t), \\
v^{\prime}\left(\lambda \sum_{i=1}^{3}\left\|z_{i}\right\|^{a}, t\right), \\
v^{\prime}\left(\lambda \sum_{i=1}^{3}\left\|z_{i}\right\|^{a_{i}}, t\right),
\end{array}\right.
\end{aligned}
$$

for all $z_{1}, z_{2}, z_{3} \in \mathscr{A}$ and all $t>0$, where $\lambda, a, a_{i}^{\prime}$ s are constants with $\lambda>0$. Then there exists a unique additive mapping $\mathscr{H}_{1}: \mathscr{A} \longrightarrow \mathscr{B}$ and unique quadratic mapping $\mathscr{H}_{2}: \mathscr{A} \longrightarrow \mathscr{B}$ such that

$$
\begin{aligned}
& \mu\left(h(z)-\mathscr{H}_{1}(z)-\mathscr{H}_{2}(z), 2 t\right) \\
& \geq\left\{\begin{array}{l}
\mu^{\prime}(\lambda,|3-p| t+|9-p| t), \\
\mu^{\prime}\left(2 \lambda|| z||^{a}, t|3-p|+t|9-p|\right), \\
\mu^{\prime}\left(\lambda\left[|| z||^{a_{1}}+|| z||^{a_{2}}\right], t|3-p|+t|9-p|\right),
\end{array}\right. \\
& v\left(h(z)-\mathscr{H}_{1}(z)-\mathscr{H}_{2}(z), 2 t\right) \\
& \leq\left\{\begin{array}{l}
\mu^{\prime}(\lambda,|3-p| t+|9-p| t), \\
\mu^{\prime}\left(2 \lambda|| z||^{a}, t|3-p|+t|9-p|\right), \\
\mu^{\prime}\left(\lambda\left[\left.|| z\right|^{a_{1}}+|| z||^{a_{2}}\right], t|3-p|+t|9-p|\right),
\end{array}\right.
\end{aligned}
$$

all $z \in \mathscr{A}$ and all $t>0$.

\section{IFBS: Stability Results: Substitution - 2}

In this section, we investigate the generalized Ulam-Hyers stability of the functional equation (1.9) in IFB space via another substituton .

Theorem 4.1. Let $\Delta \in\{1,-1\}$. Let $H: \mathscr{A} \rightarrow \mathscr{B}$ be an odd function satisfying the inequality

$$
\left.\begin{array}{l}
\mu\left(H\left(z_{1}, z_{2}, z_{3}\right), t\right) \geq \mu^{\prime}\left(\Omega_{\mu}\left(z_{1}, z_{2}, z_{3}\right), t\right) \\
v\left(H\left(z_{1}, z_{2}, z_{3}\right), t\right) \leq v^{\prime}\left(\Omega_{v}\left(z_{1}, z_{2}, z_{3}\right), t\right)
\end{array}\right\}
$$

for all $z_{1}, z_{2}, z_{3} \in \mathscr{A}$ and all $t>0$. Then there exists a unique additive mapping $\mathscr{H}_{1}: \mathscr{A} \longrightarrow \mathscr{B}$ satisfying (1.9) and

$$
\left.\begin{array}{l}
\mu\left(\mathscr{H}_{1}(z)-h(z), t\right) \geq \mu^{\prime}\left(\Omega_{\mu}^{O}(z, z, z), \frac{t}{2}|3-p|\right) \\
v\left(\mathscr{H}_{1}(z)-h(z), t\right) \leq v^{\prime}\left(\Omega_{v}^{O}(z, z, z), \frac{t}{2}|3-p|\right)
\end{array}\right\}
$$

where

$$
\left.\begin{array}{l}
\mu^{\prime}\left(\Omega_{\mu}^{O}(z, z, z), t\right) \\
=\mu^{\prime}\left(\Omega_{\mu}\left(\frac{z}{2}, \frac{z}{2}, \frac{z}{2}\right), t\right) * \mu^{\prime}\left(\Omega_{\mu}\left(\frac{z}{2}, \frac{z}{2}, \frac{z}{6}\right), t\right) \\
v^{\prime}\left(\Omega_{y}^{O}(z, z, z), t\right) \\
=v^{\prime}\left(\Omega_{v}\left(\frac{z}{2}, \frac{z}{2}, \frac{z}{2}\right), t\right) \diamond v^{\prime}\left(\Omega_{v}\left(\frac{z}{2}, \frac{z}{2}, \frac{z}{6}\right), t\right)
\end{array}\right\}
$$

for all $z \in \mathscr{A}$ and all $t>0$. Here $\Omega_{\mu}, \Omega_{v}: \mathscr{A} \times \mathscr{A} \times \mathscr{A} \longrightarrow \mathscr{C}$ are functions such that for some $0<\left(\frac{p}{3}\right)^{\Delta}<1$ with

$$
\left.\begin{array}{l}
\mu^{\prime}\left(\Omega_{\mu}\left(3^{n \Delta} z, 3^{n \Delta} z, 3^{n \Delta} z\right), t\right) \geq \mu^{\prime}\left(p^{n \Delta} \Omega_{\mu}(z, z, z), t\right) \\
v^{\prime}\left(\Omega_{v}\left(3^{n \Delta} z, 3^{n \Delta} z, 3^{n \Delta} z\right), t\right) \leq v^{\prime}\left(p^{n \Delta} \Omega_{v}(z, z, z), t\right)
\end{array}\right\}
$$

for all $z \in \mathscr{A}$ and all $t>0$ and

$$
\left.\begin{array}{l}
\lim _{n \rightarrow \infty} \mu^{\prime}\left(\Omega_{\mu}\left(3^{\Delta n} z_{1}, 3^{\Delta n} z_{2}, 3^{n \Delta} z_{3}\right), 3^{\Delta n} t\right)=1 \\
\lim _{n \rightarrow \infty} v^{\prime}\left(\Omega_{v}\left(3^{\Delta n} z_{1}, 3^{\Delta n} z_{2}, 3^{n \Delta} z_{3}\right), 3^{\Delta n} t\right)=0
\end{array}\right\}
$$

for all $z_{1}, z_{2}, z_{3} \in \mathscr{A}$ and all $t>0$.

Proof. Case (1): Let $\Delta=1$.

Using oddness of $h$ and transform $\left(z_{1}, z_{2}, z_{3}\right)$ by $\left(\frac{z}{2}, \frac{z}{2}, \frac{z}{2}\right)$ and $\left(\frac{z}{2}, \frac{z}{2}, \frac{z}{6}\right)$ in $(4.1)$, respectively, we have

$$
\left.\begin{array}{l}
\mu\left(h_{o}(3 z)+h_{o}(2 z)-h_{o}(z)-8 h_{o}\left(\frac{z}{2}\right), t\right) \\
\geq \mu^{\prime}\left(\Omega_{\mu}\left(\frac{z}{2}, \frac{z}{2}, \frac{z}{2}\right), t\right) \\
v\left(h_{o}(3 z)+h_{o}(2 z)-h_{o}(z)-8 h_{o}\left(\frac{z}{2}\right), t\right) \\
\leq v^{\prime}\left(\Omega_{v}\left(\frac{z}{2}, \frac{z}{2}, \frac{z}{2}\right), t\right)
\end{array}\right\}
$$

and

$$
\left.\begin{array}{l}
\mu\left(h_{o}(2 x)+h_{o}(z)+h_{o}(z)-8 h_{o}\left(\frac{z}{2}\right), t\right) \\
\geq \mu^{\prime}\left(\Omega_{\mu}\left(\frac{z}{2}, \frac{z}{2}, \frac{z}{6}\right), t\right) \\
v\left(h_{o}(2 x)+h_{o}(z)+h_{o}(z)-8 h_{o}\left(\frac{z}{2}\right), t\right) \\
\leq v^{\prime}\left(\Omega_{v}\left(\frac{z}{2}, \frac{z}{2}, \frac{z}{6}\right), t\right)
\end{array}\right\}
$$


for all $z \in \mathscr{A}$ and all $t>0$. It follows from (4.6), (4.7) and [(IFN5)], [(IFN11)], we arrive at

$$
\begin{aligned}
& \mu\left(h_{o}(3 x)-3 h_{o}(z), 2 t\right) \\
& \geq \mu\left(h_{o}(3 z)+h_{o}(2 z)-h_{o}(z)-8 h_{o}\left(\frac{z}{2}\right), t\right) * \\
& \quad \mu\left(h_{o}(2 z)+h_{o}(z)+h_{o}(z)-8 h_{o}\left(\frac{z}{2}\right), t\right) \\
& \geq \mu^{\prime}\left(\Omega_{\mu}\left(\frac{z}{2}, \frac{z}{2}, \frac{z}{2}\right), t\right) * \mu^{\prime}\left(\Omega_{v}\left(\frac{z}{2}, \frac{z}{2}, \frac{z}{6}\right), t\right) \\
& =\mu^{\prime}\left(\Omega_{\mu}^{O}(z), t\right) \\
& v\left(h_{o}(3 x)-3 h_{o}(z), 2 t\right) \\
& \leq \quad v\left(h_{o}(3 z)+h_{o}(2 z)-h_{o}(z)-8 h_{o}\left(\frac{z}{2}\right), t\right) \diamond \\
& \quad v\left(h_{o}(2 z)+h_{o}(z)+h_{o}(z)-8 h_{o}\left(\frac{z}{2}\right), t\right) \\
& \leq v^{\prime}\left(\Omega_{\mu}\left(\frac{z}{2}, \frac{z}{2}, \frac{z}{2}\right), t\right) \diamond v^{\prime}\left(\Omega_{v}\left(\frac{z}{2}, \frac{z}{2}, \frac{z}{6}\right), t\right) \\
& =v^{\prime}\left(\Omega_{v}^{O}(z), t\right)
\end{aligned}
$$

for all $z \in \mathscr{A}$ and all $t>0$. Using (IFN4) and (IFN10) in (4.8), we arrive

$$
\left.\begin{array}{l}
\mu\left(\frac{h(3 z)}{3}-h(z), \frac{2 t}{3}\right) \geq \mu^{\prime}\left(\Omega_{\mu}^{O}(z), t\right) \\
v\left(\frac{h(3 z)}{3}-h(z), \frac{2 t}{3}\right) \leq v^{\prime}\left(\Omega_{v}^{O}(z), t\right)
\end{array}\right\}
$$

for all $z \in \mathscr{A}$ and all $t>0$. Substituting $z$ by $3^{n} z$ in (4.9), we have

$$
\left.\begin{array}{l}
\mu\left(\frac{h\left(3^{n+1} z\right)}{3}-h\left(3^{n} z\right), \frac{2 t}{3}\right) \geq \mu^{\prime}\left(\Omega_{\mu}^{O}\left(3^{n} z\right), t\right) \\
v\left(\frac{h\left(3^{n+1} z\right)}{3}-h\left(3^{n} z\right), \frac{2 t}{3}\right) \leq v^{\prime}\left(\Omega_{v}^{O}\left(3^{n} z\right), t\right)
\end{array}\right\}
$$

for all $z \in \mathscr{A}$ and all $t>0$. It is easy to verify from (4.10) and using (4.4), (IFN4), (IFN10) that

$$
\left.\begin{array}{l}
\mu\left(\frac{h\left(3^{n+1} z\right)}{3^{(n+1)}}-\frac{h\left(3^{n} z\right)}{3^{n}}, \frac{2 t}{3 \cdot 3^{n}}\right) \geq \mu^{\prime}\left(\Omega_{\mu}^{O}(z), \frac{t}{p^{n}}\right) \\
v\left(\frac{h\left(3^{n+1} z\right)}{3^{(n+1)}}-\frac{h\left(3^{n} z\right)}{3^{n}}, \frac{2 t}{3 \cdot 3^{n}}\right) \leq v^{\prime}\left(\Omega_{v}^{O}(z), \frac{t}{p^{n}}\right)
\end{array}\right\}
$$

for all $z \in \mathscr{A}$ and all $t>0$. Interchanging $t$ into $p^{n} t$ in (4.11), we have

$$
\left.\begin{array}{l}
\mu\left(\frac{h\left(3^{n+1} z\right)}{3^{(n+1)}}-\frac{h\left(3^{n} z\right)}{3^{n}}, \frac{2 t \cdot p^{n}}{3 \cdot 3^{n}}\right) \geq \mu^{\prime}\left(\Omega_{\mu}^{O}(z), t\right) \\
v\left(\frac{h\left(3^{n+1} z\right)}{3^{(n+1)}}-\frac{h\left(3^{n} z\right)}{3^{n}}, \frac{2 t \cdot p^{n}}{3 \cdot 3^{n}}\right) \leq v^{\prime}\left(\Omega_{v}^{O}(z), t\right)
\end{array}\right\}
$$

for all $z \in \mathscr{A}$ and all $t>0$. It is easy to see that

$$
\frac{h\left(3^{n} z\right)}{3^{n}}-h(z)=\sum_{i=0}^{n-1} \frac{h\left(3^{i+1} z\right)}{3^{(i+1)}}-\frac{h\left(3^{i} z\right)}{3^{i}}
$$

for all $z \in \mathscr{A}$. From equations (4.11) and (4.13), we get

$$
\left.\begin{array}{l}
\mu\left(\frac{h\left(3^{n} z\right)}{3^{n}}-h(z), \sum_{i=0}^{n-1} \frac{2 t p^{i}}{3 \cdot 3^{i}}\right) \\
\geq \prod_{i=0}^{n-1} \mu\left(\frac{h\left(3^{i+1} z\right)}{3^{(i+1)}}-\frac{h\left(3^{i} z\right)}{3^{i}}, \frac{2 t p^{i}}{3 \cdot 3^{i}}\right) \\
v\left(\frac{h\left(3^{n} z\right)}{3^{n}}-h(z), \sum_{i=0}^{n-1} \frac{2 t p^{i}}{3 \cdot 3^{i}}\right) \\
\leq \prod_{i=0}^{n-1} v\left(\frac{h\left(3^{i+1} z\right)}{3^{(i+1)}}-\frac{h\left(3^{i} z\right)}{3^{i}}, \frac{2 t p^{i}}{3 \cdot 3^{i}}\right)
\end{array}\right\}
$$

with

$$
\prod_{i=0}^{n-1} c_{j}=c_{1} * c_{2} * \cdots * c_{n} \quad \text { and } \quad \prod_{i=0}^{n-1} d_{j}=d_{1} \diamond d_{2} \diamond \cdots \diamond d_{n}
$$

for all $z \in \mathscr{A}$ and all $t>0$. Thus

$$
\left.\begin{array}{l}
\mu\left(\frac{h\left(3^{n} z\right)}{3^{n}}-h(z), \sum_{i=0}^{n-1} \frac{2 t p^{i}}{3 \cdot 3^{i}}\right) \\
\geq \prod_{i=0}^{n-1} \mu^{\prime}\left(\Omega_{\mu}^{O}(z), t\right)=\mu^{\prime}\left(\Omega_{\mu}^{O}(z), t\right) \\
v\left(\frac{h\left(3^{n} z\right)}{3^{n}}-h(z), \sum_{i=0}^{n-1} \frac{2 t p^{i}}{3 \cdot 3^{i}}\right) \\
\leq \prod_{i=0}^{n-1} v^{\prime}\left(\Omega_{v}^{O}(z), t\right)=v^{\prime}\left(\Omega_{v}^{O}(z), t\right)
\end{array}\right\}
$$

for all $z \in \mathscr{A}$ and all $t>0$. Replacing $z$ by $3^{m} z$ in (4.15) and using (4.5), (IFN4), (IFN10), we obtain

$$
\left.\begin{array}{l}
\mu\left(\frac{h\left(3^{n+m} z\right)}{3^{(n+m)}}-\frac{h\left(3^{m} z\right)}{3^{m}}, \sum_{i=0}^{n-1} \frac{2 t p^{i}}{3 \cdot 3^{(i+m)}}\right) \\
\geq \mu^{\prime}\left(\Omega_{\mu}^{O}(z)\left(3^{m} z\right), t\right)=\mu^{\prime}\left(\Omega_{\mu}^{O}(z), \frac{t}{p^{m}}\right) \\
v\left(\frac{h\left(3^{n+m} z\right)}{3^{(n+m)}}-\frac{h\left(3^{m} z\right)}{3^{m}}, \sum_{i=0}^{n-1} \frac{2 t p^{i}}{3 \cdot 3^{(i+m)}}\right) \\
\leq v^{\prime}\left(\Omega_{v}^{O}(z)\left(3^{m} z\right), t\right)=v^{\prime}\left(\Omega_{v}^{O}(z), \frac{t}{p^{m}}\right)
\end{array}\right\}
$$

for all $z \in \mathscr{A}$ and all $t>0$ and all $m, n \geq 0$. Interchanging $t$ by $p^{m} t$ in (4.16), we get

$$
\left.\begin{array}{l}
\mu\left(\frac{h\left(3^{n+m} z\right)}{3^{(n+m)}}-\frac{h\left(3^{m} z\right)}{3^{m}}, \sum_{i=0}^{n-1} \frac{2 t p^{i+m}}{3 \cdot 3^{(i+m)}}\right) \geq \mu^{\prime}\left(\Omega_{\mu}^{O}(z), t\right) \\
v\left(\frac{h\left(3^{n+m} z\right)}{3^{(n+m)}}-\frac{h\left(3^{m} z\right)}{3^{m}}, \sum_{i=0}^{n-1} \frac{2 t p^{i+m}}{3 \cdot 3^{(i+m)}}\right) \leq v^{\prime}\left(\Omega_{v}^{O}(z), t\right)
\end{array}\right\}
$$

for all $z \in \mathscr{A}$ and all $t>0$ and all $m, n \geq 0$. Using (IFN4), (IFN10) in (4.17) implies that

$$
\left.\begin{array}{l}
\mu\left(\frac{h\left(3^{n+m} z\right)}{3^{(n+m)}}-\frac{h\left(3^{m} z\right)}{3^{m}}, t\right) \geq \mu^{\prime}\left(\Omega_{\mu}^{O}(z), \frac{t}{\sum_{i=m}^{n-1} \frac{2 p^{i}}{3 \cdot 3^{i}}}\right) \\
v\left(\frac{h\left(3^{n+m} z\right)}{3^{(n+m)}}-\frac{h\left(3^{m} z\right)}{3^{m}}, t\right) \leq v^{\prime}\left(\Omega_{v}^{O}(z), \frac{t}{\sum_{i=m}^{n-1} \frac{2 p^{i}}{3 \cdot 3^{i}}}\right)
\end{array}\right\}
$$

holds for all $z \in \mathscr{A}$ and all $t>0$ and all $m, n \geq 0$. Since $0<p<1$ and $\sum_{i=0}^{n}\left(\frac{p}{1}\right)^{i}<\infty$. The Cauchy criterion for convergence in IFNS shows that the sequence $\left\{\frac{h\left(3^{n} z\right)}{3^{n}}\right\}$ is Cauchy in $(\mathscr{B}, \mu, v)$. Since $(\mathscr{B}, \mu, v)$ is a complete IFN-space this sequence converges to some point $\mathscr{H}_{1}(z) \in \mathscr{B}$. So, one can define the mapping $\mathscr{H}_{1}: \mathscr{A} \longrightarrow \mathscr{B}$ by

$$
\begin{aligned}
& \lim _{n \rightarrow \infty} \mu\left(\frac{h\left(3^{n} z\right)}{3^{n}}-\mathscr{H}_{1}(z), t\right)=1, \\
& \lim _{n \rightarrow \infty} v\left(\frac{h\left(3^{n} z\right)}{3^{n}}-\mathscr{H}_{1}(z), t\right)=0
\end{aligned}
$$

for all $z \in \mathscr{A}$ and all $t>0$. Hence

$$
\frac{h\left(3^{n} z\right)}{3^{n}} \stackrel{I F}{\longrightarrow} \mathscr{H}_{1}(z), \quad \text { as } \quad n \rightarrow \infty
$$


Letting $m=0$ in (4.18), we arrive

$$
\left.\begin{array}{l}
\mu\left(\frac{h\left(3^{n} z\right)}{3^{n}}-h(z), t\right) \geq \mu^{\prime}\left(\Omega_{\mu}^{O}(z), \frac{t}{\sum_{i=0}^{n-1} \frac{2 p^{i}}{3 \cdot 3^{i}}}\right) \\
v\left(\frac{h\left(3^{n} z\right)}{3^{n}}-h(z), t\right) \leq v^{\prime}\left(\Omega_{v}^{O}(z), \frac{t}{\sum_{i=0}^{n-1} \frac{2 p^{i}}{3 \cdot 3^{i}}}\right)
\end{array}\right\}
$$

for all $z \in \mathscr{A}$ and all $t>0$. Letting $n$ tend to infinity in (4.19), we have

$$
\left.\begin{array}{l}
\mu\left(\mathscr{H}_{1}(z)-h(z), t\right) \geq \mu^{\prime}\left(\Omega_{\mu}^{O}(z), \frac{t}{2}(3-p)\right) \\
v\left(\mathscr{H}_{1}(z)-h(z), t\right) \leq v^{\prime}\left(\Omega_{v}^{O}(z), \frac{t}{2}(3-p)\right)
\end{array}\right\}
$$

for all $z \in \mathscr{A}$ and all $t>0$. To prove $\mathscr{H}_{1}$ satisfies (1.9) and it is unique the proof is similar to that of 3.1 .

Corollary 4.2. Assume an odd function $H: \mathscr{A} \longrightarrow \mathscr{B}$ satisfies the double inequality

$$
\begin{gathered}
\mu\left(H\left(z_{1}, z_{2}, z_{3}\right), t\right) \geq\left\{\begin{array}{l}
\mu^{\prime}(\lambda, t), \\
\mu^{\prime}\left(\lambda \sum_{i=1}^{3}\left\|z_{i}\right\|^{a}, t\right), \\
\mu^{\prime}\left(\lambda \sum_{i=1}^{3}\left\|z_{i}\right\|^{a_{i}}, t\right), \\
\mu^{\prime}\left(\lambda \prod_{i=1}^{3}\left\|z_{i}\right\|^{a}, t\right), \\
\mu^{\prime}\left(\lambda \prod_{i=1}^{3}\left\|z_{i}\right\|^{\left.a_{i}, t\right)},\right.
\end{array}\right. \\
v\left(H\left(z_{1}, z_{2}, z_{3}\right), t\right) \leq\left\{\begin{array}{l}
v^{\prime}(\lambda, t), \\
v^{\prime}\left(\lambda \sum_{i=1}^{3}\left\|z_{i}\right\|^{a}, t\right), \\
v^{\prime}\left(\lambda \sum_{i=1}^{3}\left\|z_{i}\right\|^{a_{i}}, t\right), \\
v^{\prime}\left(\lambda \prod_{i=1}^{3}\left\|z_{i}\right\|^{a}, t\right), \\
v^{\prime}\left(\lambda \prod_{i=1}^{3}\left\|z_{i}\right\|^{a_{i}}, t\right),
\end{array}\right.
\end{gathered}
$$

for all $z_{1}, z_{2}, z_{3} \in \mathscr{A}$ and all $t>0$, where $\lambda, a, a_{i}^{\prime}$ s are constants with $\lambda>0$. Then there exists a unique additive mapping

$$
\begin{aligned}
& \mathscr{H}_{1}: \mathscr{A} \longrightarrow \mathscr{B} \text { such that } \\
& \mu\left(h(z)-\mathscr{H}_{1}(z), t\right) \\
& \geq\left\{\begin{array}{l}
\mu^{\prime}(2 \lambda,|3-p| t), \\
\mu^{\prime}\left(2 \lambda|| z\left|\|^{a}\left[\frac{5}{2^{a}}+\frac{1}{6^{a}}\right], t\right| 3-p \mid\right), \\
\mu^{\prime}\left(2 \lambda\left[\frac{2 \| z||_{1}^{\mid}}{2^{a_{1}}}+\frac{2\|z \mid\|_{2}}{2^{a_{2}}}+\frac{\| z||_{3}}{2^{a_{3}}}+\frac{\|z \mid\|_{3}^{a_{3}}}{6^{a_{3}}}\right],\right. \\
t|3-p|), \\
\mu^{\prime}\left(2 \lambda|| z \|^{3 a}\left[\frac{1}{2^{3 a}}+\frac{1}{2^{2 a} a^{a}}\right], t|3-p|\right), \\
\mu^{\prime}\left(2 \lambda|| z \mid \|^{a_{1}+a_{2}+a_{3}}\left[\frac{1}{2^{a_{1}+a_{2}+a_{3}}}+\frac{1}{\left.2^{a_{1}+a_{2} \cdot 6^{a_{3}}}\right]}\right]\right. \\
t|3-p|),
\end{array}\right. \\
& v\left(h(z)-\mathscr{H}_{1}(z), t\right) \\
& \leq\left\{\begin{array}{l}
v^{\prime}(2 \lambda,|3-p| t), \\
v^{\prime}\left(2 \lambda|| z\left|\|^{a}\left[\frac{5}{2^{a}}+\frac{1}{6^{a}}\right], t\right| 3-p \mid\right), \\
v^{\prime}\left(2 \lambda\left[\frac{2\|z\|^{a_{1}}}{2^{a_{1}}}+\frac{2 \| z||_{2}}{2^{a_{2}}}+\frac{\left.\|z\|\right|_{3} ^{a_{3}}}{2^{a_{3}}}+\frac{\|z \mid\|_{3}}{6^{a_{3}}}\right],\right. \\
t|3-p|), \\
v^{\prime}\left(2 \lambda|| z \|^{3 a}\left[\frac{1}{2^{3 a}}+\frac{1}{\left.2^{2 a^{a}}\right]}\right], t|3-p|\right), \\
v^{\prime}\left(2 \lambda|| z \|^{a_{1}+a_{2}+a_{3}}\left[\frac{1}{2^{a_{1}+a_{2}+a_{3}}}+\frac{1}{\left.2^{a_{1}+a_{2} \cdot 6^{a_{3}}}\right]}\right.\right. \\
t|3-p|),
\end{array}\right.
\end{aligned}
$$

for all $z \in \mathscr{A}$ and all $t>0$.

Theorem 4.3. Let $\Delta \in\{1,-1\}$. Let $H: \mathscr{A} \rightarrow \mathscr{B}$ be an even function satisfying the inequality

$$
\left.\begin{array}{l}
\mu\left(H\left(z_{1}, z_{2}, z_{3}\right), t\right) \geq \mu^{\prime}\left(\Omega_{\mu}\left(z_{1}, z_{2}, z_{3}\right), t\right) \\
\nu\left(H\left(z_{1}, z_{2}, z_{3}\right), t\right) \leq v^{\prime}\left(\Omega_{v}\left(z_{1}, z_{2}, z_{3}\right), t\right)
\end{array}\right\}
$$

for all $z_{1}, z_{2}, z_{3} \in \mathscr{A}$ and all $t>0$. Then there exists a unique quadratic mapping $\mathscr{H}_{2}: \mathscr{A} \longrightarrow \mathscr{B}$ satisfying (1.9) and

$$
\left.\begin{array}{l}
\mu\left(\mathscr{H}_{2}(z)-h(z), t\right) \geq \mu^{\prime}\left(\Omega_{\mu}^{E}(z), t|16-p|\right) \\
v\left(\mathscr{H}_{2}(z)-h(z), t\right) \leq v^{\prime}\left(\Omega_{v}^{E}(z), t|16-p|\right)
\end{array}\right\}
$$

where

$$
\left.\begin{array}{l}
\mu^{\prime}\left(\Omega_{\mu}^{E}(z), t\right)=\mu^{\prime}\left(\Omega_{\mu}\left(\frac{z}{2}, \frac{z}{2}, \frac{z}{3}\right), t\right) \\
v^{\prime}\left(\Omega_{\mu}^{E}(z), t\right)=v^{\prime}\left(\Omega_{v}\left(\frac{z}{2}, \frac{z}{2}, \frac{z}{3}\right), t\right)
\end{array}\right\}
$$

for all $z \in \mathscr{A}$ and all $t>0$. Here $\Omega_{\mu}, \Omega_{v}: \mathscr{A} \times \mathscr{A} \times \mathscr{A} \longrightarrow \mathscr{C}$ are functions such that for some $0<\left(\frac{p}{4}\right)^{\Delta}<1$ with

$$
\left.\begin{array}{l}
\mu^{\prime}\left(\Omega_{\mu}\left(4^{n \Delta} z, 4^{n \Delta} z, 4^{n \Delta} z\right), t\right) \geq \mu^{\prime}\left(p^{n \Delta} \Omega_{\mu}(z, z, z), t\right) \\
v^{\prime}\left(\Omega_{v}\left(4^{n \Delta} z, 4^{n \Delta} z, 4^{n \Delta} z\right), t\right) \leq v^{\prime}\left(p^{n \Delta} \Omega_{v}(z, z, z), t\right)
\end{array}\right\}
$$

for all $z \in \mathscr{A}$ and all $t>0$ and

$$
\left.\begin{array}{l}
\lim _{n \rightarrow \infty} \mu^{\prime}\left(\Omega_{\mu}\left(4^{\Delta n} z_{1}, 4^{\Delta n} z_{2}, 4^{n \Delta} z_{3}\right), 16^{\Delta n} t\right)=1 \\
\lim _{n \rightarrow \infty} v^{\prime}\left(\Omega_{v}\left(4^{\Delta n} z_{1}, 4^{\Delta n} z_{2}, 4^{n \Delta} z_{3}\right), 16^{\Delta n} t\right)=0
\end{array}\right\}
$$

for all $z_{1}, z_{2}, z_{3} \in \mathscr{A}$ and all $t>0$. 
Proof. Case (1): Let $\Delta=1$.

Using evenness of $h$ and transform $\left(z_{1}, z_{2}, z_{3}\right)$ by $\left(\frac{z}{2}, \frac{z}{2}, \frac{z}{3}\right)$ in (4.23), we have

$$
\left.\begin{array}{l}
\mu\left(h_{e}(4 x)-16 h_{e}(z), t\right) \\
\geq \mu^{\prime}\left(\Omega_{\mu}\left(\frac{z}{2}, \frac{z}{2}, \frac{z}{3}\right), t\right)=\mu^{\prime}\left(\Omega_{\mu}^{E}(z), t\right) \\
v\left(h_{e}(4 x)-16 h_{e}(z), t\right) \\
\leq v^{\prime}\left(\Omega_{\mu}\left(\frac{z}{2}, \frac{z}{2}, \frac{z}{3}\right), t\right)=v^{\prime}\left(\Omega_{v}^{E}(z), t\right)
\end{array}\right\}
$$

for all $z \in \mathscr{A}$ and all $t>0$. Using (IFN4) and (IFN10) in (4.28), we arrive

$$
\left.\begin{array}{l}
\mu\left(\frac{h(4 z)}{16}-h(z), \frac{t}{16}\right) \geq \mu^{\prime}\left(\Omega_{\mu}^{E}(z), t\right) \\
v\left(\frac{h(4 z)}{16}-h(z), \frac{t}{16}\right) \leq v^{\prime}\left(\Omega_{v}^{E}(z), t\right)
\end{array}\right\}
$$

for all $z \in \mathscr{A}$ and all $t>0$. The rest of the proof is similar to that of Theorem 3.1 .

The following corollary is an immediate consequence of Theorem 3.3, regarding some stabilities of (1.9)

Corollary 4.4. Assume an even function $H: \mathscr{A} \longrightarrow \mathscr{B}$ satisfies the double inequality

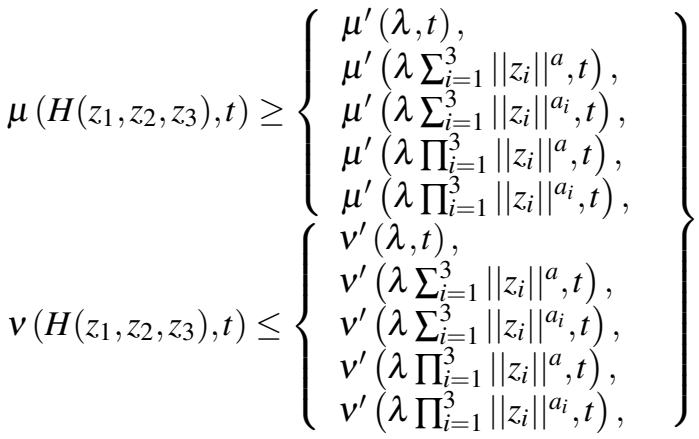

for all $z_{1}, z_{2}, z_{3} \in \mathscr{A}$ and all $t>0$, where $\lambda, a, a_{i}^{\prime}$ s are constants with $\lambda>0$. Then there exists a unique quadratic map- ping $\mathscr{H}_{2}: \mathscr{A} \longrightarrow \mathscr{B}$ such that

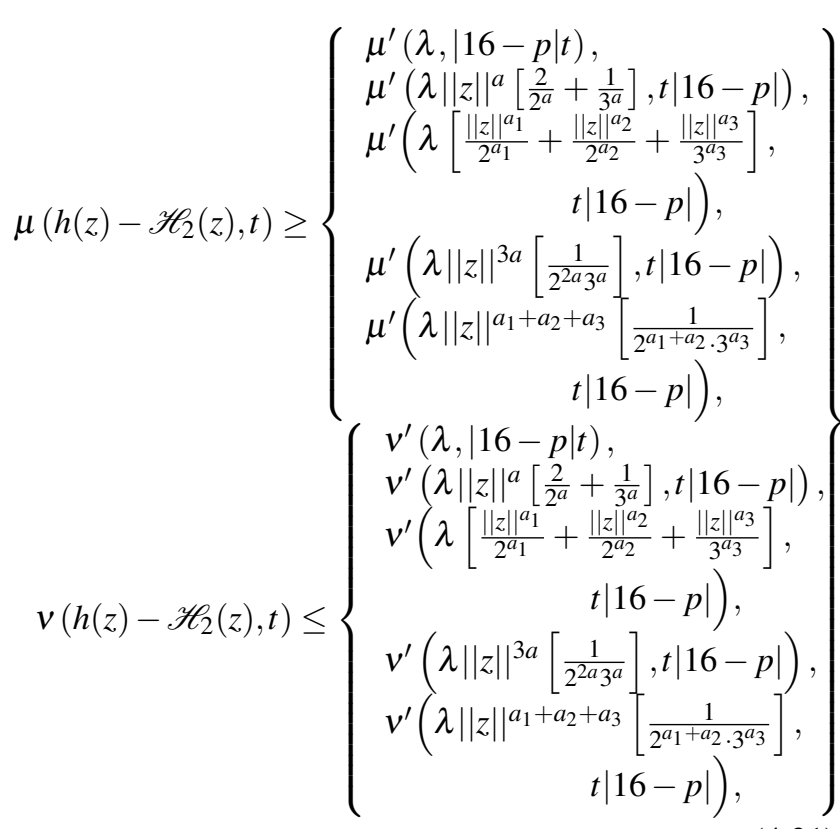

for all $z \in \mathscr{A}$ and all $t>0$.

Theorem 4.5. Let $\Delta \in\{1,-1\}$. Let $H: \mathscr{A} \rightarrow \mathscr{B}$ be a function satisfying the inequality

$$
\left.\begin{array}{l}
\mu\left(H\left(z_{1}, z_{2}, z_{3}\right), t\right) \geq \mu^{\prime}\left(\Omega_{\mu}\left(z_{1}, z_{2}, z_{3}\right), t\right) \\
\nu\left(H\left(z_{1}, z_{2}, z_{3}\right), t\right) \leq \boldsymbol{v}^{\prime}\left(\Omega_{v}\left(z_{1}, z_{2}, z_{3}\right), t\right)
\end{array}\right\}
$$

for all $z_{1}, z_{2}, z_{3} \in \mathscr{A}$ and all $t>0$. Then there exists a unique additive mapping $\mathscr{H}_{1}: \mathscr{A} \longrightarrow \mathscr{B}$ and a unique quadratic mapping $\mathscr{H}_{2}: \mathscr{A} \longrightarrow \mathscr{B}$ satisfying (1.9) and

$$
\left.\begin{array}{l}
\mu\left(h(z)-\mathscr{H}_{1}(z)-\mathscr{H}_{2}(z), t\right) \geq \mu^{\prime}\left(\Omega_{\mu}^{O E}(z), t\right) \\
v\left(h(z)-\mathscr{H}_{1}(z)-\mathscr{H}_{2}(z), t\right) \leq v^{\prime}\left(\Omega_{v}^{O E}(z), t\right)
\end{array}\right\}
$$

where

$$
\left.\begin{array}{l}
\mu^{\prime}\left(\Omega_{\mu}^{O E}(z), t\right)=\mu^{\prime}\left(\Omega_{\mu}^{O}(z)+\Omega_{\mu}^{E}(z), \frac{t}{2}|3-p|+t|16-p|\right) \\
v^{\prime}\left(\Omega_{\mu}^{O E}(z), t\right)=v^{\prime}\left(\Omega_{v}^{O}(z)+\Omega_{v}^{E}(z), \frac{t}{2}|3-p|+t|16-p|\right)
\end{array}\right\}
$$

for all $z \in \mathscr{A}$ and all $t>0$. Here $\Omega_{\mu}, \Omega_{v}: \mathscr{A} \times \mathscr{A} \times \mathscr{A} \longrightarrow \mathscr{C}$ are functions such that for some $0<\left(\frac{p}{3}\right)^{\Delta}<1 ; 0<\left(\frac{p}{4}\right)^{\Delta}<$ 1 with conditions (4.4), (4.26) and (4.5), (4.27) for all $z \in \mathscr{A}$ and all $t>0$ and for all $z_{1}, z_{2}, z_{3} \in \mathscr{A}$ and all $t>0$.

Proof. Let $h_{O}(z)=\frac{h(z)-h(-z)}{2}$ for all $z \in \mathscr{A}$. It is easy to verify that $h_{O}(0)=0$ and $h_{O}(-z)=-h_{O}(z)$ for all $z \in \mathscr{A}$. By 
definition of $h_{O}(z)$, we have

$$
\left.\begin{array}{l}
\mu\left(h_{O}\left(z_{1}, z_{2}, z_{3}\right), t\right) \\
=\mu\left(h\left(z_{1}, z_{2}, z_{3}\right)-h\left(-z_{1},-z_{2},-z_{3}\right), 2 t\right) \\
\geq \mu\left(h\left(z_{1}, z_{2}, z_{3}\right), t\right) * \mu\left(h\left(-z_{1},-z_{2},-z_{3}\right), t\right) \\
\geq \mu^{\prime}\left(\Omega_{\mu}\left(z_{1}, z_{2}, z_{3}\right), t\right) * \mu^{\prime}\left(\Omega_{\mu}\left(-z_{1},-z_{2},-z_{3}\right), t\right) \\
v\left(h_{O}\left(z_{1}, z_{2}, z_{3}\right), t\right) \\
=v\left(h\left(z_{1}, z_{2}, z_{3}\right)-h\left(-z_{1},-z_{2},-z_{3}\right), 2 t\right) \\
\leq v\left(h\left(z_{1}, z_{2}, z_{3}\right), t\right) \diamond v\left(h\left(-z_{1},-z_{2},-z_{3}\right), t\right) \\
\leq v^{\prime}\left(\Omega_{v}\left(z_{1}, z_{2}, z_{3}\right), t\right) \diamond v^{\prime}\left(\Omega_{v}\left(-z_{1},-z_{2},-z_{3}\right), t\right)
\end{array}\right\}
$$

for all $z_{1}, z_{2}, z_{3} \in \mathscr{A}$ and all $t>0$. By Theorem 4.1 , there exists a unique additive mapping $\mathscr{H}_{1}: \mathscr{A} \longrightarrow \mathscr{B}$ satisfying (1.9) and

$$
\begin{aligned}
& \mu\left(h_{O}(z)-\mathscr{H}_{1}(z), t\right) \\
& \geq \mu^{\prime}\left(\Omega_{\mu}^{O}(z), \frac{t}{2}|3-p|\right) * \mu^{\prime}\left(\Omega_{\mu}^{O}(z), \frac{t}{2}|3-p|\right) \\
& \geq \mu^{\prime}\left(\Omega_{\mu}^{O}(z), \frac{t}{2}|3-p|\right) \\
& v\left(h_{O}(z)-\mathscr{H}_{1}(z), t\right) \\
& \leq v^{\prime}\left(\Omega_{v}^{O}(z), \frac{t}{2}|3-p|\right) \diamond v^{\prime}\left(\Omega_{v}^{O}(z), \frac{t}{2}|3-p|\right) \\
& \leq v^{\prime}\left(\Omega_{v}^{O}(z), \frac{t}{2}|3-p|\right)
\end{aligned}
$$

where $\mu^{\prime}\left(\Omega_{\mu}^{O}(z), t\right)$ and $v^{\prime}\left(\Omega_{v}^{O}(z), t\right)$ are defined in (4.3) for all $z \in \mathscr{A}$ and all $t>0$.

Also, let $h_{E}(z)=\frac{h(z)+h(-z)}{2}$ for all $z \in \mathscr{A}$. It is easy to verify that $h_{E}(0)=0$ and $h_{E}(-z)=h_{E}(z)$ for all $z \in \mathscr{A}$. By definition of $h_{E}(z)$, we have

$$
\left.\begin{array}{l}
\mu\left(h_{E}\left(z_{1}, z_{2}, z_{3}\right), t\right) \\
=\mu\left(h\left(z_{1}, z_{2}, z_{3}\right)+h\left(-z_{1},-z_{2},-z_{3}\right), 2 t\right) \\
\geq \mu\left(h\left(z_{1}, z_{2}, z_{3}\right), t\right) * \mu\left(h\left(-z_{1},-z_{2},-z_{3}\right), t\right) \\
\geq \mu^{\prime}\left(\Omega_{\mu}\left(z_{1}, z_{2}, z_{3}\right), t\right) * \mu^{\prime}\left(\Omega_{\mu}\left(-z_{1},-z_{2},-z_{3}\right), t\right) \\
v\left(h_{E}\left(z_{1}, z_{2}, z_{3}\right), t\right) \\
=v\left(h\left(z_{1}, z_{2}, z_{3}\right)+h\left(-z_{1},-z_{2},-z_{3}\right), 2 t\right) \\
\leq v\left(h\left(z_{1}, z_{2}, z_{3}\right), t\right) \diamond v\left(h\left(-z_{1},-z_{2},-z_{3}\right), t\right) \\
\leq v^{\prime}\left(\Omega_{v}\left(z_{1}, z_{2}, z_{3}\right), t\right) \diamond v^{\prime}\left(\Omega_{v}\left(-z_{1},-z_{2},-z_{3}\right), t\right)
\end{array}\right\}
$$

for all $z_{1}, z_{2}, z_{3} \in \mathscr{A}$ and all $t>0$. Also, by Theorem 4.3 , there exists a unique quadratic mapping $\mathscr{H}_{2}: \mathscr{A} \longrightarrow \mathscr{B}$ satisfying (1.9) and

$$
\left.\begin{array}{l}
\mu\left(h_{E}(z)-\mathscr{H}_{2}(z), t\right) \\
\geq \mu^{\prime}\left(\Omega_{\mu}^{E}(z), t|16-p|\right) * \mu^{\prime}\left(\Omega_{\mu}^{E}(z), t|16-p|\right) \\
\geq \mu^{\prime}\left(\Omega_{\mu}^{E}(z), t|16-p|\right) \\
v\left(h_{E}(z)-\mathscr{H}_{2}(z), t\right) \\
\leq v^{\prime}\left(\Omega_{v}^{E}(z), t|16-p|\right) \diamond v^{\prime}\left(\Omega_{v}^{E}(z), t|16-p|\right) \\
\leq v^{\prime}\left(\Omega_{v}^{E}(z), t|16-p|\right)
\end{array}\right\}
$$

where $\mu^{\prime}\left(\Omega_{\mu}^{E}(z), t\right)$ and $v^{\prime}\left(\Omega_{v}^{E}(z), t\right)$ are defined in (4.25) for all $z \in \mathscr{A}$ and all $t>0$.
Suppose if we define a function $h(z)$ by

$$
h(z)=h_{O}(z)+h_{E}(z)
$$

for all $z \in \mathscr{A}$. It follows from (4.36), (4.38), (4.39), we arrive

$$
\begin{aligned}
& \mu\left(h(z)-\mathscr{H}_{1}(z)-\mathscr{H}_{2}(z), 2 t\right) \\
& =\mu\left(h_{O}(z)+h_{E}(z)-\mathscr{H}_{1}(z)-\mathscr{H}_{2}(z), 2 t\right) \\
& \geq \mu\left(h_{O}(z)-\mathscr{H}_{1}(z), t\right) * \mu\left(h_{E}(z)-\mathscr{H}_{2}(z), t\right) \\
& \geq \mu^{\prime}\left(\Omega_{\mu}^{O}(z), \frac{t}{2}|3-p|\right) * \mu^{\prime}\left(\Omega_{\mu}^{E}(z), t|16-p|\right) \\
& =\mu^{\prime}\left(\Omega_{\mu}^{O}(z)+\Omega_{\mu}^{E}(z), \frac{t}{2}|3-p|+t|16-p|\right) \\
& =\mu^{\prime}\left(\Omega_{\mu}^{O E}(z), t\right) \\
& v\left(h(z)-\mathscr{H}_{1}(z)-\mathscr{H}_{2}(z), 2 t\right) \\
& =v\left(h_{O}(z)+h_{E}(z)-\mathscr{H}_{1}(z)-\mathscr{H}_{2}(z), 2 t\right) \\
& \geq v\left(h_{O}(z)-\mathscr{H}_{1}(z), t\right) \diamond v\left(h_{E}(z)-\mathscr{H}_{2}(z), t\right) \\
& \geq v^{\prime}\left(\Omega_{v}^{O}(z), \frac{t}{2}|3-p|\right) \diamond v^{\prime}\left(\Omega_{v}^{E}(z), t|16-p|\right) \\
& =v^{\prime}\left(\Omega_{v}^{O}(z)+\Omega_{v}^{E}(z), \frac{t}{2}|3-p|+t|16-p|\right) \\
& =v^{\prime}\left(\Omega_{v}^{O E}(z), t\right)
\end{aligned}
$$

for all $z \in \mathscr{A}$ and all $t>0$.

The following corollary is an immediate consequence of Theorem 4.5, regarding some stabilities of (1.9).

Corollary 4.6. Assume a function $H: \mathscr{A} \longrightarrow \mathscr{B}$ satisfies the double inequality

$$
\begin{aligned}
\mu\left(H\left(z_{1}, z_{2}, z_{3}\right), t\right) \geq\left\{\begin{array}{l}
\mu^{\prime}(\lambda, t), \\
\mu^{\prime}\left(\lambda \sum_{i=1}^{3}\left\|z_{i}\right\|^{a}, t\right), \\
\mu^{\prime}\left(\lambda \sum_{i=1}^{3}\left\|z_{i}\right\|^{a_{i}}, t\right), \\
\mu^{\prime}\left(\lambda \prod_{i=1}^{3}\left\|z_{i}\right\|^{a}, t\right), \\
\mu^{\prime}\left(\lambda \prod_{i=1}^{3}\left\|z_{i}\right\|^{a_{i}}, t\right),
\end{array}\right. \\
v\left(H_{e}\left(z_{1}, z_{2}, z_{3}\right), t\right) \leq\left\{\begin{array}{l}
v^{\prime}(\lambda, t), \\
v^{\prime}\left(\lambda \sum_{i=1}^{3}\left\|z_{i}\right\|^{a}, t\right), \\
v^{\prime}\left(\lambda \sum_{i=1}^{3}\left\|z_{i}\right\|^{a_{i}}, t\right), \\
v^{\prime}\left(\lambda \prod_{i=1}^{3}\left\|z_{i}\right\|^{a}, t\right), \\
v^{\prime}\left(\lambda \prod_{i=1}^{3}\left\|z_{i}\right\|^{a_{i}}, t\right),
\end{array}\right\}
\end{aligned}
$$

for all $z_{1}, z_{2}, z_{3} \in \mathscr{A}$ and all $t>0$, where $\lambda, a, a_{i}^{\prime} s$ are constants with $\lambda>0$. Then there exists a unique additive mapping $\mathscr{H}_{1}: \mathscr{A} \longrightarrow \mathscr{B}$ and unique quadratic mapping $\mathscr{H}_{2}: \mathscr{A} \longrightarrow \mathscr{B}$ 
such that

$$
\begin{aligned}
& \mu\left(h(z)-\mathscr{H}_{1}(z)-\mathscr{H}_{2}(z), 2 t\right)
\end{aligned}
$$

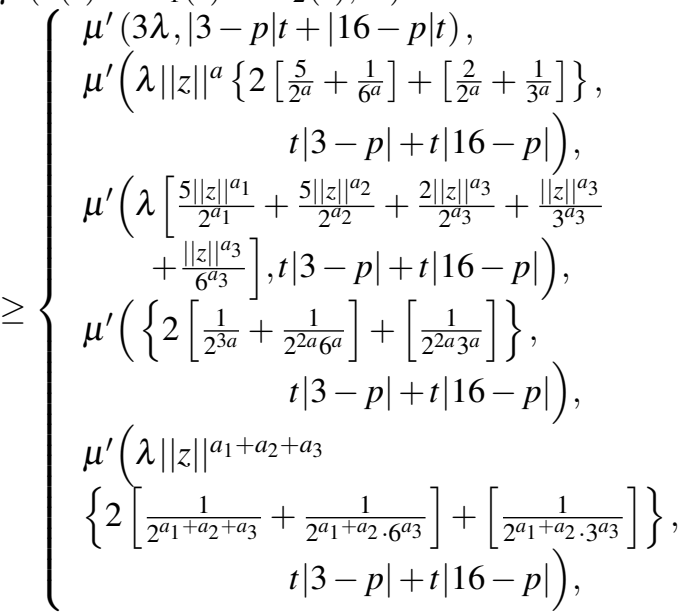

$$
\begin{aligned}
& v\left(h(z)-\mathscr{H}_{1}(z)-\mathscr{H}_{2}(z), 2 t\right) \\
& \left\{\begin{array}{l}
v^{\prime}(3 \lambda,|3-p| t+|16-p| t), \\
v^{\prime}\left(\lambda|| z||^{a}\left\{2\left[\frac{5}{2^{a}}+\frac{1}{6^{a}}\right]+\left[\frac{2}{2^{a}}+\frac{1}{3^{a}}\right]\right\}\right.
\end{array}\right. \\
& t|3-p|+t|16-p|), \\
& \mu^{\prime}\left(\lambda\|z\|^{a}\left\{2\left[\frac{5}{2^{a}}+\frac{1}{6^{a}}\right]+\left[\frac{2}{2^{a}}+\frac{1}{3^{a}}\right]\right\},\right. \\
& t|3-p|+t|16-p|),
\end{aligned}
$$

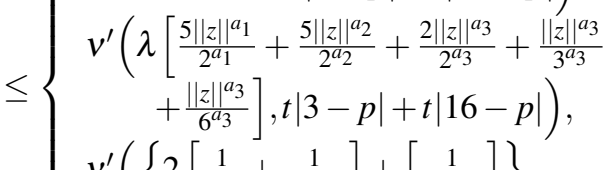

$$
\begin{aligned}
& v^{\prime}\left(\left\{2\left[\frac{1}{2^{3 a}}+\frac{1}{2^{2 a} 6^{a}}\right]+\left[\frac{1}{2^{2 a} 3^{a}}\right]\right\},\right. \\
& t|3-p|+t|16-p|), \\
& v^{\prime}\left(\lambda\|z\|^{a_{1}+a_{2}+a_{3}}\right.
\end{aligned}
$$

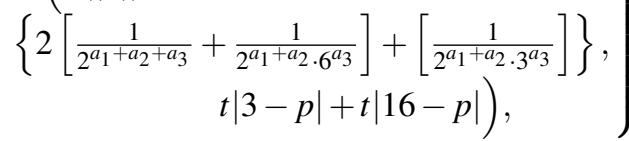

all $z \in \mathscr{A}$ and all $t>0$.

\section{References}

[1] J. Aczel and J. Dhombres, Functional Equations in Several Variables, Cambridge Univ, Press, 1989.

[2] T. Aoki, On the stability of the linear transformation in Banach spaces, J. Math. Soc. Japan 2 (1950), 64-66.

[3] M. Arunkumar, G. Ganapathy, S. Murthy, S. Karthikeyan, Stability of the Generalized Arun-additive functional equation in Intuitionistic fuzzy normed spaces, International Journal Mathematical Sciences and Engineering Applications, 4 No. V (2010), 135-146.

[4] M. Arunkumar, John M. Rassias, On the generalized Ulam-Hyers stability of an AQ- mixed type functional equation with counter examples, Far East Journal of Applied Mathematics, Volume 71, No. 2, (2012), 279-305.
[5] M. Arunkumar, P. Agilan, Additive Quadratic functional equation are Stable in Banach space: A Fixed Point Approach, International Journal of pure and Applied Mathematics, 86 No.6, (2013), 951-963.

[6] M. Arunkumar, P. Agilan, Additive Quadratic functional equation are Stable in Banach space: A Direct Method, Far East Journal of Mathematical Sciences ,80 No 1 (2013) ,105-121.

[7] M. Arunkumar, Solution and stability of modified additive and quadratic functional equation in generalized 2-normed spaces, International Journal Mathematical Sciences and Engineering Applications, Vol. 7 No. I (January, 2013), 383-391.

[8] M. Arunkumar, Perturbation of n Dimensional AQ mixed type Functional Equation via Banach Spaces and Banach Algebra: Hyers Direct and Alternative Fixed Point Methods, International Journal of Advanced Mathematical Sciences (IJAMS), Vol. 2 (1), (2014), 34-56.

[9] M. Arunkumar and S. Karthikeyan, Solution and Intuitionistic Fuzzy Stability of $n$-Dimensional Quadratic Functional Equation: Direct and Fixed Point Methods, Int. J. of Advanced Mathematical Sciences, 2 (1) (2014), 21-33.

[10] M. Arunkumar, T. Namachivayam, Intuitionistic fuzzy stability of a n-dimensional cubic functional equation: Direct and fixed point methods, Intern. J. Fuzzy Mathematical Archive, 7 (1) (2015), 1-11 .

[11] M. Arunkumar, John M. Rassias and S. Karthikeyan, Stability of a Leibniz type additive and quadratic functional equation in intuitionistic fuzzy normed spaces, Advances in theoretical and applied mathematics, 11 (2016), No.2, 145-169.

[12] K. Atanassov, Intuitionistic fuzzy sets, Fuzzy sets and Systems. 20 No. 1 (1986), 87-96.

[13] A. Bodaghi, Intuitionistic fuzzy stability of the generalized forms of cubic and quartic functional equations, J. Intel. Fuzzy Syst. 30 (2016), 2309-2317.

[14] A. Bodaghi, C. Park and J. M. Rassias Fundamental stabilities of the nonic functional equation in intuitionistic fuzzy normed spaces, Commun. Korean Math. Soc. 31, No. 4 (2016).

[15] S. Czerwik, Functional Equations and Inequalities in Several Variables, World Scientific, River Edge, NJ, 2002.

[16] G. Deschrijver, E.E. Kerre, On the relationship between some extensions of fuzzy set theory, Fuzzy Sets and Systems, 23 (2003), 227-235.

[17] M. Eshaghi Gordji, Stability of an Additive-Quadratic Functional Equation of Two Variables in F-Spaces, J. Nonlinear Sci. Appl. 2 No.4 (2009), 251-259.

[18] M.E. Gordji, N. Ghobadipour, J.M. Rassias, Fuzzy stability of additive- quadratic functional Equations, arXiv:0903.0842v1 [math.FA] 4 Mar 2009.

[19] P. Găvruta, A generalization of the Hyers-Ulam-Rassias stability of approximately additive mappings, J. Math. Anal. Appl. 184 (1994), 431-436. 
[20] S.B. Hosseini, D. O’Regan, R. Saadati, Some results on intuitionistic fuzzy spaces, Iranian J. Fuzzy Syst, 4 (2007), 53-64.

[21] S.B. Hosseini, D. O’Regan, R. Saadati, Some results on intuitionistic fuzzy spaces, Iranian J. Fuzzy Syst, 4 (2007) 53- 64.

[22] D. H. Hyers, On the stability of the linear functional equation, Proc. Natl. Acad. Sci. U.S.A. 27 (1941) 222224.

[23] D. H. Hyers, G. Isac, Th.M. Rassias, Stability of functional equations in several variables,Birkhäuser, Basel, 1998.

[24] S.M. Jung, Hyers-Ulam-Rassias Stability of Functional Equations in Mathematical Analysis, Hadronic Press, Palm Harbor, 2001.

[25] Pl. Kannappan, Functional Equations and Inequalities with Applications, Springer Monographs in Mathematics, 2009.

[26] A.K. Katsaras, Fuzzy topological vector spaces II, Fuzzy Sets and Systems, 12 (1984), 143-154.

[27] B.Margolis, J.B.Diaz, A fixed point theorem of the alternative for contractions on a generalized complete metric space, Bull. Amer. Math. Soc. 126 (1968), 305-309.

[28] S. A. Mohiuddine and Q. M. Danish Lohani, On generalized statistical convergence in intuitionistic fuzzy normed space, Chaos, Solitons Fract., 42 (1), (2009), 731-1737.

[29] M. Mursaleen, and K.J. Ansari, Stability Results in Intuitionistic Fuzzy Normed Spaces for a Cubic Functional Equation, Appl. Math. Inf. Sci. 7, No. 5, (2013), 16771684.

[30] M. Mursaleen and S. A. Mohiuddine, On stability of a cubic functional equation in intuitionistic fuzzy normed spaces, Chaos, Solitons and Fractals, 42 (2009), 29973005 .

[31] A. Najati, M.B. Moghimi, On the stability of a quadratic and additive functional equation, J. Math. Anal. Appl. 337 (2008), 399-415.

[32] J. H. Park, Intuitionistic fuzzy metric spaces, Chaos, Solitons and Fractals, 22 (2004), 1039-1046.

[33] M.J. Rassias, M. Arunkumar and S. Ramamoorthi, Stability of the Leibniz additive-quadratic functional equation in quasi- $\beta$ normed spaces: direct and fixed point methods, Journal of Concrete and Applicable Mathematics, 14 (2014), 22 - 46.

[34] J. M. Rassias, On approximately of approximately linear mappings by linear mappings, J. Funct. Anal. 46 (1982), 126-130.

[35] J. M. Rassias, M. Arunkumar, E.sathya, N. Mahesh Kumar, Generalized Ulam - Hyers Stability Of A (AQQ): Additive - Quadratic - Quartic Functional Equation, Malaya Journal of Matematik, 5(1) (2017), 122-142.

[36] Th. M. Rassias, On the stability of the linear mapping in Banach spaces, Proc.Amer.Math. Soc. 72 (1978), 297300.

[37] Th. M. Rassias, Functional Equations, Inequalities and
Applications, Kluwer Acedamic Publishers, Dordrecht, Bostan London, 2003.

[38] K. Ravi, M. Arunkumar, J.M. Rassias, On the Ulam stability for the orthogonally general Euler-Lagrange type functional equation, International Journal of Mathematical Sciences, 3 (2008), 36-47.

[39] R. Saadati, J. H. Park, On the intuitionistic fuzzy topological spaces, Chaos, Solitons and Fractals. 27 (2006), 331-344.

[40] R. Saadati, J. H. Park, Intuitionstic fuzzy Euclidean normed spaces, Commun. Math. Anal., 1 (2006), 85-90.

[41] R. Saadati, S. Sedghi and N. Shobe, Modified intuitionistic fuzzy metric spaces and some fixed point theorems, Chaos, Solitons and Fractals, 38 (2008), 36-47.

[42] S.Shakeri, Intuitionstic fuzzy stability of Jensen type mapping, J. Nonlinear Sci. Appli. 22 (2009), 105-112.

[43] S. M. Ulam, Problems in Modern Mathematics, Science Editions, Wiley, New York, 1964.

[44] L. A. Zadeh, Fuzzy sets, Inform. Control, 8 (1965), 338-353.

[45] D. X. Zhou, On a conjecture of Z. Ditzian, J. Approx. Theory, 69 (1992), 167-172.

[46] S. Zolfaghari, Approximation Of Mixed Type Functional Equations In $p$-Banach Spaces, J. Nonlinear Sci. Appl. 3 (2010), no. 2, 110-122.

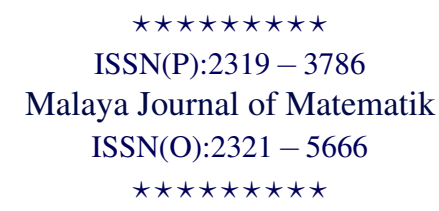

